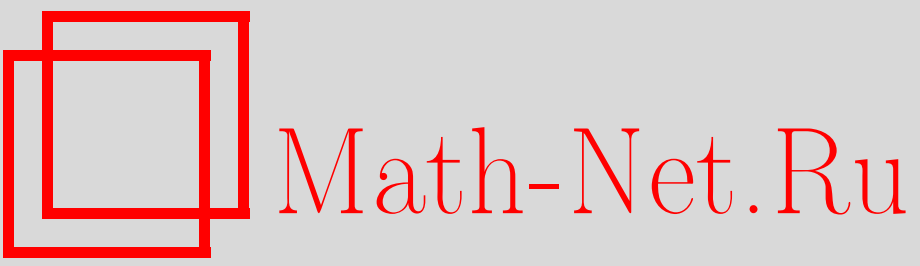

О. Е. Барндорф-Нильсен, Ю. Шмигель, Пространственно-временное моделирование, основанное на процессах Леви, и его приложения к турбулентности, УМH, 2004, том 59, выпуск 1, 63-90

DOI: https://doi.org/10.4213/rm701

Использование Общероссийского математического портала Math-Net.Ru подразумевает, что вы прочитали и согласны с пользовательским соглашением

http://www.mathnet.ru/rus/agreement

Параметры загрузки:

IP : 3.85 .7 .115

26 апреля 2023 г., 17:41:01 


\section{ПРОСТРАНСТВЕННО-ВРЕМЕННОЕ МОДЕЛИРОВАНИЕ, ОСНОВАННОЕ НА ПРОЦЕССАХ ЛЕВИ, И ЕГО ПРИЛОЖЕНИЯ К ТУРБУЛЕНТНОСТИ}

О. Е. БАРНДОРФ-НИЛЬСЕН, Ю. ШМИГЕЛЬ

В статье рассматриваются некоторые типы пространственно-временных моделей, построенных с помощью базисов Леви. Динамика описьвается полем стохастических процессов $X=\left\{X_{t}(\sigma)\right\}$ (на множестве $\mathscr{S}$ узлов $\sigma$ ), определенных интегралами

$$
X_{t}(\sigma)=\int_{-\infty}^{t} \int_{\mathscr{S}} f_{t}(\rho, s ; \sigma) Z(\mathrm{~d} \rho \times \mathrm{d} s),
$$

где $Z$ обозначает базис Леви. Подынтегральные выражения $f$ являются детерминированными функциями вида $f_{t}(\rho, s ; \sigma)=h_{t}(\rho, s ; \sigma) \mathbf{1}_{A_{t}(\sigma)}(\rho, \sigma)$, где $h_{t}(\rho, s ; \sigma)$ имеет специальный вид и $A_{t}(\sigma)$ - подмножество в $\mathscr{S} \times \mathbb{R}_{\leqslant t}$.

Мы сначала рассмотрим ОУ-поля (поля Орнштейна-Уленбека) $X_{t}(\sigma)$, которые являются обобшением понятия ОУ-процессов (процессов типа Орнштейна-Уленбека), сосредоточиваясь в основном на возможностях процесса $X_{t}(\sigma)$ для динамического моделирования. Кратко указьваются приложения к динамическим пространственным процессам типа Кокса.

Во второй части статьи обсуждается моделированиепространственно-временных корреляций СП-полей $(\mathrm{CП} \mathrm{=} \mathrm{стохастическая} \mathrm{прерывистость)} \mathrm{вида}$

$$
Y_{t}(\sigma)=\exp \left\{X_{t}(\sigma)\right\}
$$

Этот вид позволяет явно вычислить математические ожидания

$$
\mathrm{E}\left\{Y_{t_{1}}\left(\sigma_{1}\right) \cdots Y_{t_{n}}\left(\sigma_{n}\right)\right\}
$$

которыемы используем при характеризации корреляций. СП-поля могут рассматриваться как динамическое, непрерывное и однородное обобщение турбулентных каскадов. В этой связи мы строим СП-поле с пространственно-временным масштабньдм поведением, согласованным с диссипацией энергии, наблюдаемой в турбулентных потоках. Кратко обсуждаются некоторые параллели к этому построению.

Библиография: 53 названия.

\section{СОДЕРЖАНИЕ}

$\S 1$. Введение

$\S 2$. Основания 


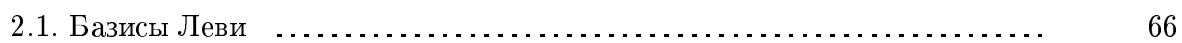

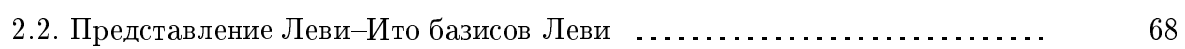

2.3. Интегралы по отношению к базисам Леви _....................... 68

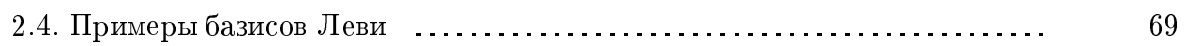

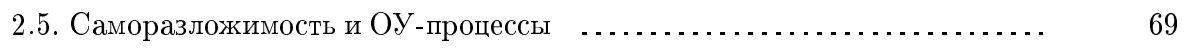

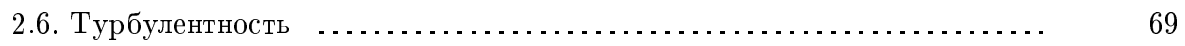

§ 3. Пространственно-временные процессы ОУ-типа ................. 71

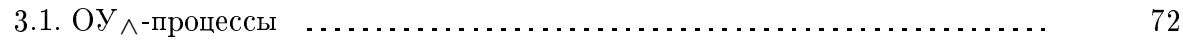

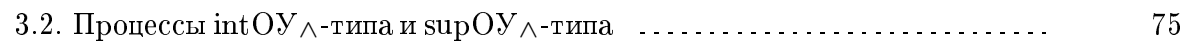

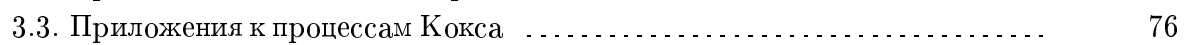

3.4. Расширение ............................................ 77

3.5. О пространственной структуре ОУ $\wedge$-процессов ..................

$\S 4$. Пространственно-временные процессы СП-типа . .................. 78

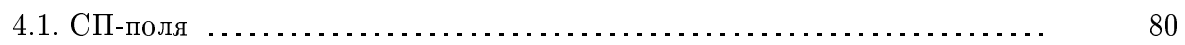

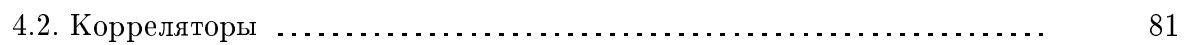

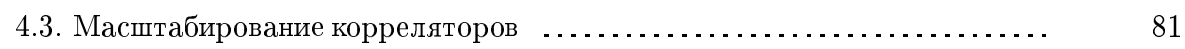

4.4. Далшнейшее моделирование двухточечных корреляторов ............. 84

4.5. Динамическое среднедисперсное моделирование . . . . . . . . . . . . . . . . . 85

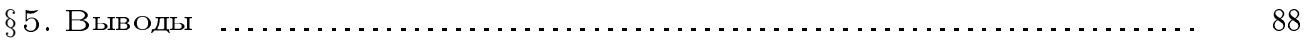

Список литературы . . . . . .

\section{$\S 1$. Введение}

Понятие базисов Леви является богатым источником пространственно-временно́го моделирования, и эти базисы приводят к разным новшествам, например, в соответствующих моделях Орнштейна-Уленбека и моделях для масштабирующего поведения в турбулентности. В настоящей статье указаны некоторые приложения этого понятия. В частности, обсуждаются связи с недавними важными достижениями в моделировании турбулентности с точки зрения мультипликативных каскадов.

Мы будем рассуждать в терминах множества узлов $\mathscr{S}$ с точками $\sigma$, где события развиваются с течением времени $t$. Динамическое развитие будет описываться полем стохастических процессов $X=\left\{X_{t}(\sigma)\right\}$ на $\mathscr{S}$, которые будут определяться интегралами вида

$$
X_{t}(\sigma)=\int_{-\infty}^{t} \int_{\mathscr{S}} f_{t}(\rho, s ; \sigma) Z(\mathrm{~d} \rho \times \mathrm{d} s),
$$

где $Z$ означает базис Леви, т.е. безгранично делимую и независимо рассеянную случайную меру на $\mathscr{R}=\mathscr{S} \times \mathbb{R}$. Для конкретности, в настоящей статье предполагается, что подынтегральные выражения $f$ являются неслучайными функциями, а $\mathscr{S}$ - борелевское подмножество $\mathbb{R}^{d}$. С каждой точкой $\xi=(\sigma, t)$ в $\mathscr{R}$ мы связываем множество $A_{t}(\sigma)$ в $\mathscr{B}(\mathscr{R})$, где $\mathscr{B}(\mathscr{R})$ означает борелевскую $\sigma$-алгебру на $\mathscr{R}$, и рассматриваем функции $f$ вида

$$
f_{t}(\rho, s ; \sigma)=h_{t}(\rho, s ; \sigma) \mathbf{1}_{A_{t}(\sigma)}(\rho, s),
$$

где $h$ подчиняется различным ограничениям, например, $h_{t}(\rho, s ; \sigma)=\exp (-\lambda(\sigma)(t-s))$ или $h_{t}(\rho, s ; \sigma)=h(|\rho-\sigma|)$, где $|\cdot|$ означает расстояние в $\mathbb{R}^{d}$. Мы назьваем $A_{t}(\sigma)$ множествами размаха. 
Далее в основном будут рассматриваться два случая, которые мы назьваем ОУ-полями и СП-полями соответственно. В обоих случаях множества $A_{t}(\sigma)$ относятся к прошльм событиям в том смысле, что $A_{t}(\sigma) \cap\left(\mathbb{R}^{d} \times(t, \infty)\right)=\varnothing$. Прототип множеств $A_{t}(\sigma)$ для ОУ-полей изображен на рис. 1 , а прототип множеств $A_{t}(\sigma)$ для СП-полейна рис. 2.

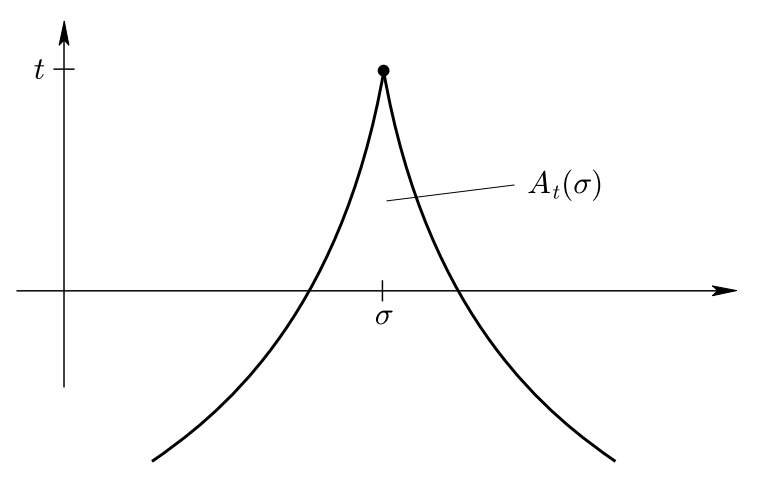

Рис. 1. Пример множества размаха $A_{t}(\sigma)$, связанного с $X_{t}(\sigma)$

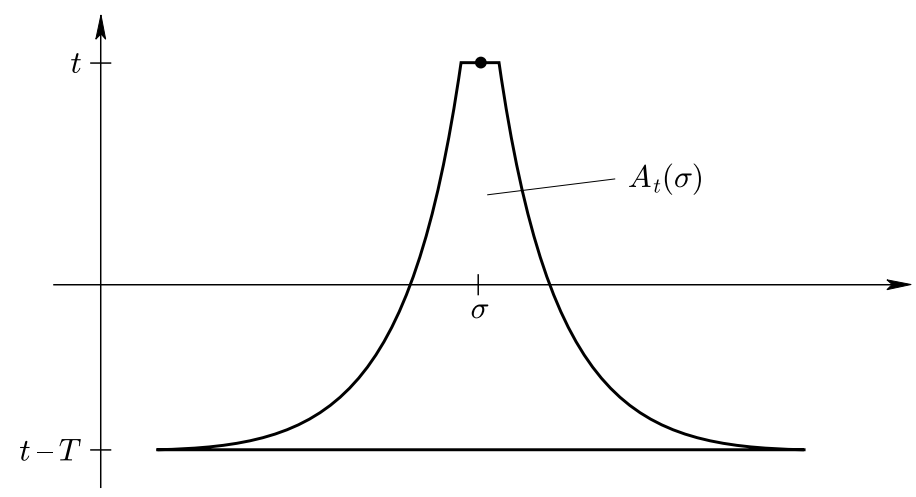

Рис. 2. Пример множества размаха $A_{t}(\sigma)$, связанного с $Y_{t}(\sigma)$

ОУ-поля являются обобшением понятия ОУ-процессов (процессов типа Орнштейна-У Ленбека). Обшее обсуждение этих процессов можно найти в [44]. В ряде недавних работ по финансовой математике ([9], [10], [39]; см. также [45] и [13]) положительные одномерные ОУ-процессы были успешно использованы как модели стохастической изменчивости (волатильности) финансовых активов (некоторая работа в многомерном случае проделана в [7]). Существенное сходство между динамикой финансовых рынков и динамикой турбулентных жидкостей (см., например, [1], [4], [26]) вызывает интерес к реалистическому моделированию прерывистости, которая является турбулентным аналогом стохастической изменчивости, с помошю идей, связанных с 
ОУ-процессами. Как мы увидим, эти идеи могут быть также полезны в других областях пространственно-временного моделирования, например, при моделировании процессов напряженности для применения в динамических пространственных процессах типа Кокса.

Мотивация для введения СП-полей (СП - стохастическая прерьвистость) связана с недавними успехами моделирования турбулентных каскадов с точки зрения мультипликативных процессов [49] (В этой связи см. также [27].)

В этой статье мы кратко приводим ряд результатов об ОУ- и СП-полях, оставляя много математических деталей до более полного изложения в другом месте. Параграф 2 содержит основные сведения о моделировании, использующем базисы Леви, и о турбулентности. В $\S \S 3$ и 4 рассматриваются ОУ- и СП-поля соответственно. ОУ-поля имеют линейньй тип $X_{t}(\sigma)$ (как в $(1)$ ), а СП-поля являются полями мультипликативного типа, т.е. экспоненциированньми версиями $Y_{t}(\sigma)=\exp \left\{X_{t}(\sigma)\right\}$. Отдельньй интерес представляют интегралы от $X_{t}(\sigma)$ и от $Y_{t}(\sigma)$ по переменной $\sigma{ }^{1}$ В этой статье мы в основном сосредоточиваемся на масштабируюшем поведении диссипации энергии (закон $\frac{4}{5}$ и его модификации), не рассматривая чаще изучаемые масштабируюшие особенности поля скоростей (закон $\frac{n}{3}$ и его модификации) и спектр мощности (закон $\frac{5}{3}$ и его модификации), которые будут обсуждаться в $[8]^{2}$. В разделе 4.3 мы рассматриваем динамические модели полей диссипации энергии с особым вниманием к временным двумерным корреляторам. В разделах 4.4 и 4.5 кратко обсуждается моделирование дальнейших аспектов СП-полей. Мы снова сосредоточиваемся на временном моделировании, но во многих случаях можно перевести результаты в пространственные соотношения. Параграф 5 носит заключительный характер.

\section{$\S$ 2. Основания}

В настоящем параграфе суммированы некоторые хорошо известные результаты по теории безграничной делимости и процессов Леви, а также по теории турбулентности. Они служат основньм материалом в рассуждениях в оставшейся части работы.

Мы используем следующие обозначения для лог-характеристической функции и для лог-преобразования Лапласа случайной переменной $X$ :

$$
\mathrm{C}\{\zeta \neq X\}=\ln \mathrm{E}\left\{e^{i \zeta X}\right\} \text { и } \mathrm{K}\{\theta \ddagger X\}=\ln \mathrm{E}\left\{e^{\theta X}\right\}
$$

и, чтобы различать их, будем назьвать их с-кумулянтной функцией и k-кумулянтной функцией соответственно. Аналогичные обозначения будут использоваться для векторных случайных величин и условных законов. Так, например, С $\{\zeta \ddagger X \mid Y\}-$ условная с-кумулянтная функция для $X$ при данном $Y$.

2.1. Базисы Леви. Обозначим через $\mathscr{B}=\mathscr{B}(\mathscr{R})$ борелевскую $\sigma$-алгебру в $\mathscr{R}=$ $\mathscr{S} \times \mathbb{R}$, и пусть символ $\mathscr{B}_{b}$ обозначает класс ограниченных элементов в $\mathscr{B}$. Пусть

\footnotetext{
${ }^{1}$ Последний тип интегралов позволяет нам понять, что может быть названо экспоненциальными функционалами на основе базисов Леви. В этой связи см. [21].

2 десь, однако, уместно сослаться на работу [20], в которой вводятся и изучаются процессы полей скоростей типа дробового шума, с особым вниманием к моделированию среднемасштабных структур в океанских волнах.
} 
$Z=\{Z(A) ; A \in \mathscr{C}\}$, где $\mathscr{C}=\mathscr{B}$ или $\mathscr{B}_{b},-$ независимо рассеянная случайная мера на $\mathscr{R}$.

Предположим, что $Z$ безгранично делима в том смысле, что $Z(A)$ - безгранично делимая случайная величина, с-кумулянтная функция которой может быть представлена в виде

$$
\mathrm{C}\{\zeta \ddagger Z(A)\}=i \zeta a(A)-\frac{1}{2} \zeta^{2} b(A)+\int_{\mathbb{R}}\left\{e^{i \zeta x}-1-i \zeta \mathbf{1}_{[-1,1]}(x)\right\} \nu(\mathrm{d} x, A)
$$

для любого $A \in \mathscr{C}$, где $a$ - мера со знаком (заряд) на $\mathscr{B}, b$ - положительная мера на $\mathscr{B}$, а $\nu(\mathrm{d} x, A)$ - мера Леви на $\mathbb{R}$ при фиксированном $A$ и мера на $\mathscr{B}$ при фиксированном $\mathrm{d} x$.

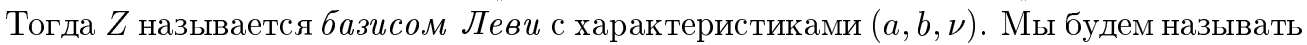
$\nu$ обобщенной мерой Леви. В этой статье нас будут интересовать только случаи, в которых гауссовой части нет, т.е. $b$ всюду равно 0 . Тогда управляющая мера базиса $Z$ имеет вид

$$
\omega(\mathrm{d} \xi)=|a|(\mathrm{d} \xi)+\int_{\mathbb{R}}\left(x^{2} \wedge 1\right) \nu(\mathrm{d} x, \mathrm{~d} \xi) .
$$

Если базис Леви $Z$ на $\mathscr{R}$ таков, что $Z(A)$ пуассоновски распределен для любого $A$, то мы назьваем $Z$ пуассоновским базисом. В этом случае обобшенная мера Леви имеет вид $\nu(\mathrm{d} x, A)=\Lambda(A) \delta_{1}(\mathrm{~d} x)$ для некоторой меры $\Lambda$ на $\mathscr{B}$, где $\delta_{1}-$ мера Дирака в точке 1 . Однако нас больше интересуют случаи, когда $\nu$ - диффузная мера на $\mathbb{R} \times \mathscr{R}$.

Эвристически, полезно выразить (2) по-другому, в инфинитезимальном виде в $\xi \in \mathscr{R}$, а именно,

$$
\mathrm{C}\{\zeta \ddagger Z(\mathrm{~d} \xi)\}=i a(\mathrm{~d} \xi)-\frac{1}{2} \zeta^{2} b(\mathrm{~d} \xi)+\int_{\mathbb{R}}\left\{e^{i \zeta x}-1-i \zeta \mathbf{1}_{[-1,1]}(x)\right\} \nu(\mathrm{d} x ; \mathrm{d} \xi) .
$$

Мы не слишком потеряем в обшности, предполагая, что мера $\nu$ в (3) факторизуется по формуле

$$
\nu(\mathrm{d} x ; \mathrm{d} \xi)=U(\mathrm{~d} x ; \xi) \mu(\mathrm{d} \xi)
$$

для некоторой меры $\mu$ на $\mathscr{R}$, где $U(\mathrm{~d} x ; \xi)$ - мера Леви при каждом фиксированном $\xi$.

В большинстве случаев, которые мы будем рассматривать, мера Леви $U(\cdot ; \xi)$ абсолютно непрерывна относительно лебеговой меры на $\mathscr{R}$ с некоторой плотностью $u(\mathrm{~d} x ; \xi)$ при каждом $\xi$.

Мы можем считать величину

$$
\kappa(\zeta ; \xi)=\int_{\mathbb{R}}\left\{e^{i \zeta x}-1-i \zeta \mathbf{1}_{[-1,1]}(x)\right\} U(\mathrm{~d} x ; \xi)
$$

с-кумулянтной функцией некоторой случайной величины, скажем, $Z^{\prime}(\xi)$, имеющей с-кумулянтную функцию

$$
\kappa(\zeta ; \xi)=\mathrm{C}\left\{\zeta \ddagger Z^{\prime}(\xi)\right\}
$$

если $a(\mathrm{~d} \xi) \equiv 0$, то

$$
\mathrm{C}\{\zeta \ddagger Z(\mathrm{~d} \xi)\}=\mathrm{C}\left\{\zeta \ddagger Z^{\prime}(\xi)\right\} \mu(\mathrm{d} \xi)=\kappa(\zeta ; \xi) \mu(\mathrm{d} \xi)
$$


Во многих случаях, представляюших прикладной интерес, величина $U(\cdot ; \xi)$ не зависит от $\xi$. Тогда мы говорим, что базис Леви факторизуем, и пишем $U(\mathrm{~d} x)$ и т. д.

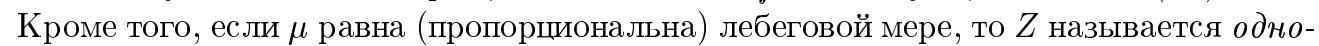
родным базисом. Если это условие вьполняется, то в качестве $Z^{\prime}$ мы берем случайную величину с мерой Леви $U$.

Вьшеприведенные рассмотрения непосредственно распространяются на многомерные базисы Леви $Z=\left(Z_{1}, \ldots, Z_{m}\right)$, и инфинитезимальное представление Леви-Хинчина принимает вид

$$
\mathrm{C}\{\zeta \ddagger Z(\mathrm{~d} \xi)\}=i\langle a(\mathrm{~d} \xi), \zeta\rangle-\frac{1}{2}\langle\zeta b(\mathrm{~d} \xi), \zeta\rangle+\int\left\{e^{i\langle\zeta, x\rangle}-1-i \zeta \mathbf{1}_{[-1,1]}(x)\right\} \nu(\mathrm{d} x ; \mathrm{d} \xi),
$$

где $\zeta=\left(\zeta_{1}, \ldots, \zeta_{m}\right), a-m$-мерная мера, а $b-(m \times m)$-матричнозначная мера.

2.2. Представление Леви-Ито базисов Леви. Пусть $Z$ - независимо рассеянная случайная мера на $\mathbb{R}^{k}$ с характеристической тройкой $(a, 0, \nu)$ и неатомической управляющей мерой $\omega$. Тогда (доказательство приведено в [41]) существует такой пуассонов базис $N$ на $\mathbb{R} \times \mathbb{R}^{k}$ с мерой интенсивности $\nu$, что $Z$ можно представить в виде

$$
Z(\mathrm{~d} \xi)=a(\mathrm{~d} \xi)+\int_{|x|>1} x N(\mathrm{~d} x ; \mathrm{d} \xi)+\int_{|x| \leqslant 1} x(N-\nu)(\mathrm{d} x ; \mathrm{d} \xi) .
$$

Если $Z$ - неотрицательный базис Леви, то это представление можно переписать в виде

$$
Z(\mathrm{~d} \xi)=a_{0}(\mathrm{~d} \xi)+\int_{\mathbb{R}_{+}} x N(\mathrm{~d} x ; \mathrm{d} \xi)
$$

для некоторой меры $a_{0}$ на $\mathbb{R}^{k}$.

2.3. Интегралы по отношению к базисам Леви. Интеграл от измеримой функции $f$ на $\mathscr{R}$ относительно базиса Леви $Z$ обозначим через $f \bullet Z$. (О теории интегрирования относительно независимо рассеянной случайной меры см. [30] или [34]. В [42] рассматривается обший вопрос о стохастическом интегрировании относительно компенсированной пуассоновой случайной меры.)

Ключевой результат для многих вычислений состоит в формуле

$$
\mathrm{C}\{\zeta \neq f \bullet Z\}=f \bullet a+\int \kappa(\zeta f(\xi) ; \xi) \mu(\mathrm{d} \xi) .
$$

Существенное условие справедливости этой формулы состоит в том, что интеграл в правой части должен существовать (ср. [14] и [8]).

Интегралы от неотрицательных функцй $f$ по неотрицательному базису Леви с обобшенной мерой Леви $\nu(d x ; d \xi)$ можно представить в виде

$$
f \bullet Z=f \bullet a_{0}+\int_{\mathbb{R}_{+}} \int_{\mathscr{S}} f(\xi) x N(\mathrm{~d} x ; \mathrm{d} \xi),
$$

и k-кумулянтная функция базиса $Z$ имеет вид

$$
\mathrm{K}\{\theta f \ddagger Z\}=\mathrm{K}\{\theta \ddagger f \bullet Z\}=\theta f \bullet a_{0}+\int_{\mathbb{R}_{+}} \int_{\mathscr{S}}\left(e^{\theta f(\xi) x}-1\right) \nu(\mathrm{d} x ; \mathrm{d} \xi) .
$$

Кроме того, мера Леви интеграла $f \bullet Z$ равна $U_{f}=\bar{f} \circ \nu$, т.е. лифтингу $\nu$ с помошью $\bar{f}$, где $\bar{f}(x, \xi)=x f(\xi)$. 


\section{4. Примеры базисов Леви.}

ПримеР 2.1 (ТУ базисы Леви). Если $U(\mathrm{~d} x ; \xi)$ в $(4)$ сосредоточена на $\mathbb{R}_{>0}$ и имеет вид

$$
U(\mathrm{~d} x ; \xi)=x^{-1-\alpha / 2} e^{-\frac{1}{2} \gamma^{2}(\xi) x} \mathrm{~d} x
$$

с $0<\alpha<2$ и $\gamma(\xi)>0$, то мы будем говорить, что $Z$ образует темперированный (умеренныц ет $\alpha / 2$ темперированное устойчивое распределение, и обратный гауссов закон получается при $\alpha=1$. (Темперированные устойчивые (TY) законы и процессы Леви обсуждаются в [11].) Закон распределения для $Z^{\prime}(\xi)$ переходит в предельном случае $\alpha=0$ в гамма-распределение $\Gamma\left(1, \frac{1}{2} \gamma^{2}(\xi)\right)$.

Если $\gamma$ не зависит от $\xi$, то базис $Z$ факторизуем, и $Z(A)$ подчиняется $T Y$ закону (гамма-закону при $\alpha=0$ ) для всех $A$.

Для дальнейшего важно, что есть естественное обобщение семейства $T Y$ законов на так называемое обобщенное гамма-семейство (или $G$-семейство), см. [17] и имеюшиеся там ссылки.

ПримеР 2.2 (НОГ базис Леви). Если $U(\mathrm{~d} x ; \xi)$ в (4) имеет вид

$$
U(\mathrm{~d} x ; \xi)=\pi^{-1} \delta \alpha(\xi)|x|^{-1} K_{1}(\alpha(\xi)|x|) e^{\beta(\xi) x}
$$

$($ с $\alpha(\xi)>|\beta(\xi)| \geqslant 0)$, то мы будем говорить, что $Z$ образует нормальный обратный гауссов (НОГ) базис Леви. Если $\alpha$ и $\beta$ не зависят от $\xi$, то $Z$ факторизуем и $Z(A)$ удовлетворяет НОГ закону для всех $A$.

2.5. Саморазложимость и ОУ-процессы. Стационарньй стохастический про-

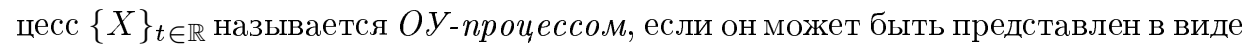

$$
X_{t}=\int_{-\infty}^{t} e^{-\lambda(t-s)} \mathrm{d} Z_{\lambda s}
$$

где $\lambda$ - положительньй параметр, так назьваемый параметр интенсивности ОУ процесса, а $Z_{t}$ - процесс Леви. В этом случае закон $X_{t}$ необходимо саморазложим, и, с другой стороны, для любого саморазложимого закона $D$ на $\mathbb{R}$ существует процесс Леви $Z$, так называемый управляюший фоновый процесс Леви (УФПЛ), такой, что формула (11) определяет ОУ-процесс $X_{t}$, распределенный в соответствии с $D$. Об общей теории саморазложимости и ОУ-процессов см. [44]. Приложения этих понятий к финансам и турбулентности обсуждаются в [1]-[4], [9], [10]; см. также [45], [13], [5], [6].

2.6. Турбулентность. Одной из основных особенностей турбулентных потоков является прерьвистое поведение поля скоростей и связанная с этим прерывистость диссипации энергии [25], [38]. Здесь прерьвистость означает, что флуктуации около среднего значения осуществляются взрьвами с амплитудами, которые явно негауссовы. Кроме того, эти прерьвистые взрьвы группируются, показывая свои временны́е зависимости. Эти характеристические особенности турбулентного потока могут также быть описаны с помощью мультифрактального формализма, где моменты разностей скоростей на данном интервале времени и моменты крупнозернистой диссипации 
энергии с заданным размером области крупнозернистости демонстрируют масштабируюшее поведение на некотором определенном промежутке, назьваемом инерциальной областью [35], [52]. Первое систематическое описание масштабирования инерциальной области принадлежит Колмогорову [31], [32]. Он использовал два основных предположения об универсальности турбулентного потока при больших числах Рейнольдса. Первое состоит в том, что если вязкость жидкости $\nu$ мала, то средняя скорость диссипации энергии $\epsilon$ независима от $\nu$. Второе предположение состоит в том, что маломасштабная турбулентность однородна и изотропна. Согласно вьводу Колмогорова, в этом случае прирашения скорости $\Delta v_{r} \equiv v(\sigma+r)-v(\sigma)$ для расстояний $r$ в пределах инерциальной области подчиняются масштабируюшему соотношению

$$
\mathrm{E}\left\{\Delta v_{r}^{n}\right\} \propto(r \epsilon)^{n / 3} .
$$

Много усилий было приложено, чтобы проверить это уравнение, и особенно его спектральный эквивалент в случае $n=2$,

$$
\Phi(\kappa) \propto \epsilon^{2 / 3} \kappa^{-5 / 3},
$$

где $\Phi(\kappa)$ - одномерный спектр энергии. Оказалось, что монофрактальное масштабирование приращений скорости не соответствует экспериментальньм наблюдениям для порядков $n>3$ (см. обзор и дальнейшие ссылки в [25], [52]). Одна из причин этого несоответствия - сильная изменяемость скорости диссипации энергии. Еще в 1962 г. А.М. Обухов [40] и А.Н. Колмогоров [33] предложили заменить $\epsilon$ локально крупнозернистой скоростью диссипации энергии

$$
\epsilon_{r}=\frac{1}{|V|} \int_{V} \epsilon(\sigma) \mathrm{d} \sigma
$$

где $|V|=O\left(r^{3}\right)$ - объем линейного размера $r$. Согласно этому предложению, сами моменты локально усредненной диссипации энергии проявляют масштабируюшее поведение с нелинейными (т.е. мультифрактальньми) показателями масштабирования $\tau(n)$,

$$
\mathrm{E}\left\{\epsilon_{r}^{n}\right\} \propto|V|^{-\tau(n)} .
$$

Предполагается, что это масштабируюшее соотношение вьполняется внутри инерциальной области (некоторого вполне определенного интервала для $r$ ) для потоков с очень высоким числом Рейнольдса. Особенно трудно выделить масштабируюшие экспоненты $\tau(n)$ для высокого порядка $n$ (и необходимо конечных чисел Рейнольдса).

Однако в [49], [48] показано, что $n$-точечные корреляторы (определенные ниже) тоже демонстрируют масштабирующее поведение, которое (по сравнению с крупнозернистой диссипацией энергии) гораздо более точно, даже для малых чисел Рейнольдса. Предположим, что соотношение $\mathrm{E}\left\{\epsilon_{r}^{2}\right\} \propto r^{-\tau(2)}$ выполняется в точности на некоторой области переменного $r$. Тогда мы можем дважды продифференцировать это соотношение и получить $\mathrm{E}\{\epsilon(\sigma) \epsilon(\sigma+r)\} \propto r^{-\tau(2)}$, поскольку

$$
\mathrm{E}\left\{\epsilon_{r}^{2}\right\}=2 \int_{0}^{r}(r-\sigma) \mathrm{E}\{\epsilon(0) \epsilon(\sigma)\} \mathrm{d} \sigma,
$$


если мы предполагаем транслящионную инвариантность. Таким образом, масштабирование двухточечных коррелящий более фундаментально в том смысле, что точное масштабирование локально усредненной диссипации энергии вызьвает масштабирование корреляторов. Но обратное утверждение может не вьполняться. Это - очень важньй факт, и он является причиной того, что мы сосредоточиваемся на масштабируюшем соотношении для $n$-точечных корреляторов в разделе 4.3 .

В настоящее время масштабируюшее описание турбулентного потока (в терминах приращений скорости или в терминах диссипации энергии) не объяснено из динамики Навье-Стокса; однако есть исключение, а именно, знаменитый колмогоровский закон 4/5 [31], [32] (исторические данные приведены в [51]). Именно по этой причине феноменологические модели играют важную роль в описании турбулентного поведения [25], [15]. Один из простейших и одновременно наиболее успешных типов таких моделей - каскадные модели, которые способны описать прерьвистое масштабируюшее поведение диссипации энергии ясным и аналитически обрабатьваемьм образом. Эти модели могут также воспроизводить наблюдаемые кратные распределения и их корреляционные эффекты, с-кумулянты и марковские свойства [46], [29], [22], [24], [48]. Несмотря на этот замечательный успех, такие модели имеют два главных недостатка. Если они определены в соответствии с каскадной структурой дерева, то они не трансляионно инвариантны, что приводит к дискретному пространственному разложению. С другой стороны, если модели определены без учета древоподобной структуры, то их корреляционная структура должна вводиться вручную. В обоих случаях трудно дать простое и полное описание каскадного процесса. Второй недостатокстатический характер моделей. Турбулентность - динамическое явление, и оно безусловно требует одновременного временно́го и пространственного описания. В контексте масштабируюших процессов остается неясным, как рационально включить в рассмотрение динамику. Таким образом, есть очевидная необходимость динамического обобшения каскадных процессов, сохраняющего их свойство гнездовой структуры переплетаюшихся масштабов. На этом пути СП-поля кажутся естественньм способом ввести динамику в мультипликативньй процесс взаимодействуюших масштабов, как обсуждается ниже в $\S 4$. В качестве примера мы обсуждаем масштабируюшие соотношения для $n$-точечных корреляторов в разделе 4.3. Мы не обсуждаем маргинальное распределение диссипации энергии, поскольку некоторые особенности этого распределения зависят от потока. В связи с универсальностью, масштабируюшие показатели $\tau(n)$ в крупнозернистой диссипации энергии и масштабируюшие показатели $n$-точечных корреляторов являются подходяшими наблюдаемыми. Однако в более детальных приближениях обшие рамки СП-полей позволяют провести дополнительное моделирование маргинальных характеристик.

\section{§ 3. Пространственно-временные процессы ОУ-типа}

В этом параграфе мы рассматриваем расширение идеи ОУ-процессов на случай пространственно-временных контекстов. Мы начинаем с обсуждения простейшего, основного типа таких процессов. С этой целью в качестве области $\mathscr{S}$ мы рассматриваем все $d$-мерное евклидово пространство $\mathbb{R}^{d}$ и предполагаем, что семейство множеств размаха транслящионно инвариантно, т.е. $A_{t}(\sigma)=(\sigma, t)+A_{0}(0)$. Мы также 
предполагаем для простоты, что базис Леви имеет меру Леви вида

$$
\nu(\mathrm{d} x, \mathrm{~d}(\sigma, t))=U(\mathrm{~d} x) \mathrm{d} \sigma \mathrm{d} t
$$

так что $Z$ однороден.

3.1. ОУ $\wedge$-процессы. Рассмотрим двупараметрическое семейство подмножеств $\mathbb{R}^{d}$ вида

$$
\left\{C_{s}(\sigma):-\infty<s \leqslant 0, \sigma \in \mathbb{R}^{d}\right\}
$$

с $C_{0}(\sigma)=\{\sigma\}$ и

$$
C_{s}(\sigma) \subset C_{s^{\prime}}(\sigma) \text { для } 0>s>s^{\prime} .
$$

В терминах этого семейства мы можем определить транслящионно инвариантные множества размаха $A_{t}(\sigma)$ по правилу

$$
A_{t}(\sigma)=\left\{(\rho, s):-\infty<s \leqslant t, \rho \in C_{s-t}(\sigma)\right\}
$$

Для краткости мы будем писать $C_{s}$ вместо $C_{s}(0)$. Таким образом, $C_{s}(\sigma)=(\sigma, t)+C_{s}$. При $s>0$ мы положим $C_{s}=C_{-s}$.

При некоторых мягких условиях, наложенных на множества $C_{s}$, мы можем теперь определить случайное поле (процесс) $X$ на $\mathscr{S}=\mathbb{R}^{d}$ по формуле

$$
X_{t}(\sigma)=\int_{-\infty}^{t} e^{-t+s} Z\left(C_{s-t}(\sigma) \times \mathrm{d} s\right) .
$$

Назовем $X_{t}(\sigma) O Y_{\wedge}$-процессом. Выражение для $X_{t}(\sigma)$ можно представить в виде

$$
X_{t}(\sigma)=\int_{-\infty}^{0} e^{u} Z\left(C_{u}(\sigma) \times \mathrm{d}_{t} u\right)
$$

где $\mathrm{d}_{t} u$ обозначает инфинитезимальньй элемент $\mathrm{d} u$ в точке $t$ временной оси. (Мы аналогичным образом используем обозначение $\mathrm{d}_{\sigma} \rho$.)

Заметим также, что при фиксированном $t$ можно рассматривать $Z\left(C_{s-t}(\sigma) \times \mathrm{d} s\right)$ как $\mathrm{d} Z^{(\sigma, t)}(s)$, где $Z^{(\sigma, t)}-$ аддитивньй процесс на $(-\infty, t]$, и тогда

$$
X_{t}(\sigma)=\int_{-\infty}^{t} e^{-t+s} \mathrm{~d} Z^{(\sigma, t)}(s)
$$

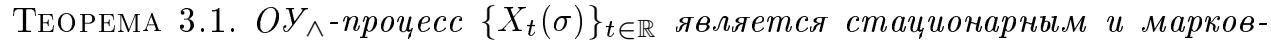
ским. При $t \geqslant 0$ положим

$$
U_{t}(\sigma)=e^{-t} \int_{-\infty}^{0} e^{s} Z\left(C_{s-t}(\sigma) \backslash C_{s}(\sigma) \times \mathrm{d} s\right)
$$

$$
V_{t}(\sigma)=e^{-t} \int_{0}^{t} e^{s} Z\left(C_{s-t}(\sigma) \times \mathrm{d} s\right)
$$




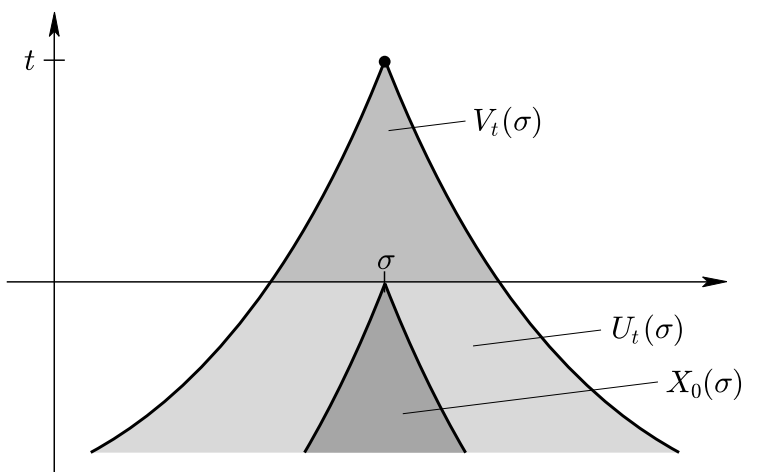

Рис. 3. Иллюстрация вкладов процессов $V_{t}(\sigma)$ и $U_{t}(\sigma)$

Тогда $\left\{X_{t}(\sigma)\right\}_{t \geqslant 0}$ допускает разложение

$$
X_{t}(\sigma)=e^{-t} X_{0}(\sigma)+U_{t}(\sigma)+V_{t}(\sigma),
$$

әде $X_{0}(\sigma),\left\{U_{t}(\sigma)\right\}_{t \geqslant 0} u\left\{V_{t}(\sigma)\right\}_{t \geqslant 0}$ независимы.. (См. рис. 3 как иллюстрацию.)

ЗАмЕчАнИЕ. В частности, из разложения (18) следует, что

$$
\mathrm{C}\left\{\zeta \ddagger X_{t}(\sigma) \mid X_{0}(\sigma)\right\}=e^{-t} X_{0}(\sigma)+\mathrm{C}\left\{\zeta \ddagger U_{t}(\sigma)\right\}+\mathrm{C}\left\{\zeta \ddagger V_{t}(\sigma)\right\} .
$$

Это показывает, что процесс $\left\{X_{t}(\sigma)\right\}_{t \in \mathbb{R}}$ аффинныц с с том смысле, что условная с-кумулянтная функция является аффинной функцией обусловливаюшего состояния. Марковские процессы, удовлетворяюшие подобному условию, были недавно изучены в большой обшности в [23].

ДоКАЗАТЕЛЬСТвО. По определению

$$
X_{0}(\sigma)=\int_{-\infty}^{0} e^{s} Z\left(C_{s}(\sigma) \times \mathrm{d} s\right) .
$$

Далее, используя (13), мы видим, что

$$
\begin{aligned}
X_{t}(\sigma)= & e^{-t} \int_{-\infty}^{0} e^{s} Z\left(C_{s-t}(\sigma) \times \mathrm{d} s\right)+e^{-t} \int_{0}^{t} e^{s} Z\left(C_{s-t}(\sigma) \times \mathrm{d} s\right) \\
= & e^{-t} \int_{-\infty}^{0} e^{s} Z\left(C_{s}(\sigma) \times \mathrm{d} s\right)+e^{-t} \int_{-\infty}^{0} e^{s} Z\left(C_{s-t}(\sigma) \backslash C_{s}(\sigma) \times \mathrm{d} s\right) \\
& +e^{-t} \int_{0}^{t} e^{s} Z\left(C_{s-t}(\sigma) \times \mathrm{d} s\right)
\end{aligned}
$$

при $t>0$, т.е.

$$
X_{t}(\sigma)=e^{-t} X_{0}(\sigma)+U_{t}(\sigma)+V_{t}(\sigma) .
$$

Поскольку $Z$ независимо рассеяна, величина $\left\{Z\left(C_{s}(\sigma) \times \mathrm{d} s\right): s \leqslant 0\right\}$ независима от

$$
\left\{Z\left(C_{s-t}(\sigma) \backslash C_{s}(\sigma) \times \mathrm{d} s\right): s \leqslant 0\right\} .
$$


Отсюда следует, что $X_{0}(\sigma),\left\{U_{t}(\sigma)\right\}_{t \in \mathbb{R}}$ и $\left\{V_{t}(\sigma)\right\}_{t \in \mathbb{R}}$ независимы и $X_{t}(\sigma)$ - марковский процесс. Стационарность $\left\{X_{t}(\sigma)\right\}_{t \in \mathbb{R}}$ немедленно следует из однородности $Z$ и транслящионной инвариантности множеств размаха.

Так как $C_{s}(\sigma)=C_{-s}(\sigma)$ по договоренности, то

или, что то же,

$$
U_{t}(\sigma) \stackrel{\text { law }}{=} \int_{t}^{\infty} e^{-u} Z\left(C_{u}(\sigma) \backslash C_{u-t}(\sigma) \times \mathrm{d} u\right)
$$

$$
U_{t}(\sigma) \stackrel{\text { law }}{=} e^{-t} Q_{t}(\sigma),
$$

где

$$
Q_{t}(\sigma)=\int_{0}^{\infty} e^{-v} Z\left(C_{v+t}(\sigma) \backslash C_{v}(\sigma) \times \mathrm{d} v\right),
$$

a $Q_{t}-$ аддитивный процесс ${ }^{3}$. Кроме того,

$$
V_{t}(\sigma) \stackrel{\text { law }}{=} V_{t}^{\uparrow}(\sigma),
$$

где

$$
V_{t}^{\uparrow}(\sigma)=\int_{0}^{t} e^{-v} Z\left(C_{v}(\sigma) \times \mathrm{d} v\right),
$$

и $V_{t}^{\uparrow}$ - тоже аддитивный процесс.

Теорема 3.2. Прочесс $\left\{X_{t}^{\uparrow}(\sigma)\right\}_{t \in \mathbb{R}_{\geqslant 0}}$, определенный формулой

$$
X_{t}^{\uparrow}(\sigma)=e^{-t} X_{0}(\sigma)+e^{-t} Q_{t}(\sigma)+V_{t}^{\uparrow}(\sigma),
$$

имеет в точности тот же закон, что $u\left\{X_{t}(\sigma)\right\}_{t \in \mathbb{R}_{\geqslant 0}}$. Здесь $Q_{t}(\sigma) u V_{t}^{\uparrow}(\sigma)-$ аддитивные процессы и

$$
\begin{aligned}
& \mathscr{L}\left(X_{0}(\sigma),\left\{U_{t}(\sigma)\right\}_{t \in \mathbb{R}_{\geqslant 0}},\left\{V_{t}(\sigma)\right\}_{t \in \mathbb{R}_{\geqslant 0}}\right) \\
& \quad=\mathscr{L}\left(X_{0}(\sigma),\left\{e^{-t} Q_{t}(\sigma)\right\}_{t \in \mathbb{R}_{\geqslant 0}},\left\{V_{t}^{\uparrow}(\sigma)\right\}_{t \in \mathbb{R}_{\geqslant 0}}\right) .
\end{aligned}
$$

Из (19) следует, что $X_{t}^{\uparrow}(\sigma)$ является решением стохастического дифференциального уравнения

$$
\begin{aligned}
\mathrm{d} X_{t}^{\uparrow}(\sigma) & =-X_{t}^{\uparrow}(\sigma) \mathrm{d} t+\mathrm{d} V_{t}^{\uparrow}(\sigma)+e^{-t} V_{t}^{\uparrow}(\sigma) \mathrm{d} t \\
& =-X_{t}^{\uparrow}(\sigma) \mathrm{d} t+e^{-t}\left(V_{t}^{\uparrow}(\sigma) \mathrm{d} t+Z\left(C_{t}(\sigma) \times \mathrm{d} t\right)\right),
\end{aligned}
$$

и, следовательно, процесс квадратической вариации для $\left\{X_{t}^{\uparrow}(\sigma)\right\}_{t \in \mathbb{R} \geqslant 0}$ задается формулой

$$
\mathrm{d}\left[X^{\uparrow}(\sigma)\right]_{t}=e^{-2 t} Z^{2}\left(C_{t}(\sigma) \times \mathrm{d} t\right) .
$$

Как шаг по направлению к моделированию различных типов временного поведения полей, мы рассматриваем теперь временнь́е дилатации процесса (14), получая процессы следующего более общего вида:

$$
X_{t}(\sigma)=\int_{-\infty}^{t} e^{-\lambda(\sigma)(t-s)} Z\left(C_{s-t}(\sigma) \times \lambda(\sigma) \mathrm{d} s\right) .
$$

\footnotetext{
${ }^{3}$ Напомним, что аддитивный процесс является процессом с независимыми приращениями. Об обобщении понятия аддитивности на многомерньй случай см. [41].
} 
Пример 3.1. Особенно простой случай возникает, если $\mathscr{S}=\mathbb{R}, \lambda=\lambda(\sigma)$ не зависит от $\sigma$ и $C_{s}(\sigma)=\sigma+[-g(s), g(s)]$ для некоторой неотрищательной убьвающей функции $g$ на $(-\infty, 0]$. Тогда

$$
X_{t}(\sigma)=e^{-\lambda t} \int_{-\infty}^{t} e^{\lambda s} Z([-g(s-t)+\sigma, g(s-t)+\sigma] \times \lambda \mathrm{d} s) .
$$

Выражения для совместных с-кумулянтов процесса $X_{t}(\sigma)$ (рассматриваемых как случайное поле на $\mathbb{R} \times \mathbb{R}$ ) могут быть легко получены из формулы $(24)$.

Как частичное обобщение этой постановки, представляет интерес задача, в которой $g(s)=c|s|$ для некоторой постоянной $c>0$, если взять $X_{t}(\sigma)$ равным

$$
X_{t}(\sigma)=\int_{-\infty}^{t} \int_{-c|s-t|+\sigma}^{c|s-t|+\sigma} e^{-\lambda|(\sigma, t),(\rho, s)|} Z(\mathrm{~d} \rho \times \lambda \mathrm{d} s),
$$

где $\mid \cdot, \cdot$ | обозначает евклидово расстояние.

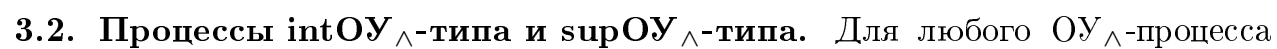
$\left\{X_{t}(\sigma)\right\}_{t \in \mathbb{R}}$ положим

$$
X_{t}^{*}(\sigma)=\int_{0}^{t} X_{s}(\sigma) \mathrm{d} s
$$

и будем назьвать этот процесс int $O y_{\wedge}$-процессом.

$\mathrm{B}$ финансовой математике аналогичное понятие intOУ-процесса является центральным в описании ключевого понятия интегрированной изменчивости. См., например, [12], [13]. Другой тип приложений указан в следующем разделе 3.3.

Чтобы прояснить природу $X_{t}^{*}(\sigma)$, используем представление (19) для процесса $X_{t}^{\uparrow}(\sigma)$, эквивалентного $X_{t}(\sigma)$. Так как $Q_{t}(\sigma)$ и $V_{t}^{\uparrow}(\sigma)$ - аддитивные процессы, то (в очевидных обозначениях)

$$
\begin{aligned}
X_{t}^{\uparrow *}(\sigma)=\int_{0}^{t} X_{s}^{\uparrow}(\sigma) \mathrm{d} s & =\left(1-e^{-t}\right) X_{0}(\sigma)+\int_{0}^{t} e^{-s} Q_{s}(\sigma) \mathrm{d} s+\int_{0}^{t} V_{s}^{\uparrow}(\sigma) \mathrm{d} s \\
& =\left(1-e^{-t}\right) X_{0}(\sigma)-e^{-t} Q_{t}(\sigma)+\int_{0}^{t} e^{-s} \mathrm{~d} Q_{s}(\sigma)+V_{t}^{\uparrow *}(\sigma) \\
& =X_{0}^{\uparrow}(\sigma)-X_{t}^{\uparrow}(\sigma)+\int_{0}^{t} e^{-s} \mathrm{~d} Q_{s}(\sigma)+V_{t}^{\uparrow}(\sigma)+V_{t}^{\uparrow *}(\sigma) .
\end{aligned}
$$

Это представление показьвает, что совместньй процесс $\left(X_{t}^{\uparrow}(\sigma), X_{t}^{\uparrow *}(\sigma)\right)$ - аффинньй, и поэтому то же верно для $\left(X_{t}(\sigma), X_{t}^{*}(\sigma)\right)$.

Заметим также, что

$$
\int_{0}^{t} e^{-s} Q_{s}(\sigma) \mathrm{d} s=\int_{0}^{t}\left(e^{-s}-e^{-t}\right) \mathrm{d} Q_{s}(\sigma)
$$

и

$$
V_{t}^{\uparrow *}(\sigma)=\int_{0}^{t}(t-s) \mathrm{d} V_{s}^{\uparrow}(\sigma)
$$


и поэтому

$$
X_{\sigma}^{\uparrow *}(\sigma)=\left(1-e^{-t}\right) X_{0}(\sigma)+\int_{0}^{t}\left(e^{-s}-e^{-t}\right) \mathrm{d} Q_{s}(\sigma)+\int_{0}^{t}(t-s) \mathrm{d} V_{s}(\sigma) .
$$

Отсюда, в частности, следует, что условное с-кумулянтное преобразование $X_{t}^{*}(\sigma)$ при данном $X_{0}(\sigma)$, которое важно для предсказаний, может быть представлено в виде

$$
\begin{aligned}
C\left\{\zeta \ddagger X_{t}^{*}(\sigma) \mid X_{0}(\sigma)\right\}= & \left(1-e^{-t}\right) X_{0}(\sigma)+\int_{0}^{t} C\left\{\left(e^{-s}-e^{-t}\right) \zeta \ddagger Z^{\prime}\right\} q(s) \mathrm{d} s \\
& +\int_{0}^{t} C\left\{(t-s) \zeta \ddagger Z^{\prime}\right\}\left|C_{s}(\sigma)\right| \mathrm{d} s
\end{aligned}
$$

где

$$
q(s)=\int_{0}^{\infty} e^{-v} \frac{\mathrm{d}\left|C_{s+v}(\sigma)\right|}{\mathrm{d} s} \mathrm{~d} v
$$

(в предположении, что $\left|C_{s+v}\right|$ диффференцируема).

Кроме того, предполагая для простоты, что базис Леви $Z$ неотрицателен, мы видим согласно (25), что значение $X_{t}^{*}(\sigma)$ во многом определяется $V_{t}^{*}(\sigma)$ для больших $t$.

$\mathrm{C}$ помощью суперпозиций независимых ОУ ${ }_{\wedge}$-процессов с различными весовыми функциями $\lambda(\sigma)$ можно ввести широкий спектр структур зависимости. Эти процессы называются supОУ $\wedge$-процессами. Мы в особенности имеем в виду их временные зависимости. Аналогичный подход был использован в финансах, см. [10], [13]. Построение использует дополнительньй базис Леви, ср. [3].

3.3. Приложения к процессам Кокса. Приложения специальных типов базисов Леви (гамма, обратных гауссовых, темперированных устойчивых) были использованы в [16], [17] и [53] в чисто пространственном контексте для моделирования меры интенсивности для процессов Кокса. Пространственные зависимости вводятся перемешиванием вида $m(A)=\int k(A, \xi) Z(\mathrm{~d} \xi)$ с подходящим образом выбранными ядрами $k$. Получаемая случайная мера $m$ снова безгранично делима и поэтому определяет процессы $m_{t}$ (случайных мер) с независимьми приращениями. Этот факт был использован в [18] для моделирования роста сорняков с помощью процессов Кокса, где поверхности интенсивности развиваются со временем в соответствии с подходяшим процессом $m_{t}$ этого рода, а лежаший в основе базис Леви принадлежит типу $G$ (ср. пример 2.1). См. также [36].

Другой подход был использован для моделирования пространственной интенсивности $\lambda$ в виде $\lambda(\xi)=\exp \{G(\xi)\}$, где $G(\xi)$ образуют гауссово случайное поле, см. [37]. Пространственно-временное обобщение этого подхода, использующее гауссово случайное поле (процесс) $G_{t}(\xi)$, развивающееся во времени в соответствии со стохастическим дифференциальньм уравнением Орнштейна-Уленбека, рассмотрено в [19].

Альтернативно, любой неотрищательньй supOУ $\wedge$-процесс (поле) $\left\{X_{t}(\cdot)\right\}_{t \in \mathbb{R}}$ может быть использован как процесс интенсивности динамического процесса Кокса на $\mathbb{S}$, и при этом интегрированньй процесс (поле) $\left\{X_{t}^{*}(\cdot)\right\}_{t \in \mathbb{R}}$ может служить как пространственная замена времени. Преимушество этого подхода по сравнению с гауссовой спецификацией лог-интенсивности в том, что обновления линейны. 
3.4. Расширение. Обобщая $(14)$, определим $X_{t}(\sigma)$ формулой

$$
X_{t}(\sigma)=e^{-\lambda(\sigma) t} \int_{-\infty}^{t} e^{\lambda(\sigma) s} \int_{\mathscr{R}} f_{s-t}(\rho ; \sigma) Z(\mathrm{~d} \rho \times \lambda(\sigma) \mathrm{d} s)
$$

где $\lambda(\sigma)>0$ - параметр временно́й дилатации, а функция $f_{s}(\rho ; \sigma)$ определена при $s \leqslant 0$ и $(\rho, \sigma) \in \mathbb{R}^{d} \times \mathbb{R}^{d}$, неотрицательна и удовлетворяет условию

$$
f_{s^{\prime}}(\rho ; \sigma) \geqslant f_{s}(\rho ; \sigma)
$$

при $0 \geqslant s>s^{\prime}$. Это условие выполняется, в частности, если $f_{s}(\rho ; \sigma)=h(\rho ; \sigma) \mathbf{1}_{C_{s}(\sigma)}(\rho)$ для некоторой (неотрицательной) функции $h(\rho ; \sigma)$, а множества $C_{s}(\sigma)$ выбраны так же, как в разделе 3.1 .

В этом случае мы снова имеем разложение

$$
X_{t}(\sigma)=e^{-\lambda(\sigma) t} X_{0}(\sigma)+U_{t}(\sigma)+V_{t}(\sigma)
$$

где теперь

$$
\begin{aligned}
& U_{t}(\sigma)=e^{-\lambda(\sigma) t} \int_{-\infty}^{0} e^{\lambda(\sigma) s} \in t_{\mathscr{R}}\left\{f_{s-t}(\rho ; \sigma)-f_{s}(\rho ; \sigma)\right\} Z(\mathrm{~d} \rho \times \lambda(\sigma) \mathrm{d} s), \\
& V_{t}(\sigma)=e^{-\lambda(\sigma) t} \int_{0}^{t} e^{\lambda(\sigma) s} \int_{\mathscr{R}} f_{s-t}(\rho ; \sigma) Z(\mathrm{~d} \rho \times \lambda(\sigma) \mathrm{d} s),
\end{aligned}
$$

и это, вместе с (26), показьвает, что $X_{t}(\sigma)$ - марковский процесс. Кроме того, $X_{t}(\sigma)$ можно также представить в виде

$$
X_{t}(\sigma)=\int_{-\infty}^{0} e^{\lambda(\sigma) s} f_{s}(\rho ; \sigma) Z\left(\mathrm{~d} \rho \times \lambda(\sigma) \mathrm{d}_{t} s\right),
$$

и отсюда следует, что $X_{t}(\sigma)$ - стационарньй процесс.

3.5. О пространственной структуре ОУ ${ }_{\wedge}$-процессов. Мы обсудили вьше некоторые свойства ОУ ${ }_{\wedge}$-процессов, а именно, временное поведение $X_{t}(\sigma)$, где $X_{t}(\sigma)$ имеет вид (14). Здесь мы кратко обсудим пространственное поведение, а именно, будем рассматривать $X_{t}(\sigma)$ как стохастический процесс с одномерной величиной $\sigma$, играющей роль времени.

Из построения, очевидно, следует, что $\left\{X_{t}(\sigma)\right\}_{\sigma \in \mathbb{R}}-$ стационарньй процесс для любого фиксированного $t$. Чтобы упростить обозначения, положим $t=0$. Предположим для простоты, что $C_{s}(0)=[-c|s|, c|s|]$ для некоторого $c>0$; тогда можно разложить $\left\{X_{0}(\sigma)\right\}_{\sigma \geqslant 0}$ с помошью аддитивных процессов следующим образом (см. рис. 4 как иллюстрацию):

$$
X_{0}(\sigma)=X_{0}(0)-X_{0}^{\searrow}(\sigma)+X_{0}^{\rightarrow}(\sigma)-X_{0}^{\downarrow}(\sigma),
$$




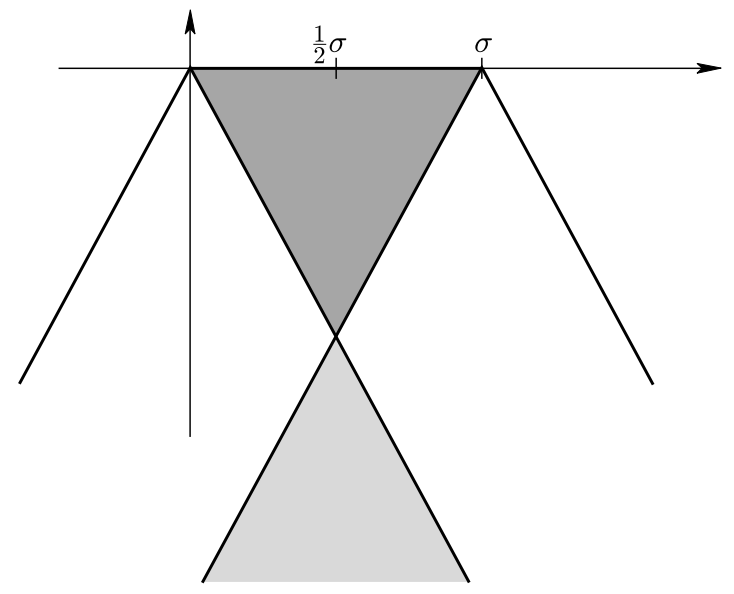

Рис. 4. Иллюстрация разложения (27)

где $X_{0}^{\searrow}(\sigma), X_{0} \rightarrow(\sigma)$ и $X_{0}^{\downarrow}(\sigma)$ - аддитивные процессы, определенные формулами

$$
\begin{aligned}
& X_{0}^{\searrow}(\sigma)=X_{0}(0)-\int_{-\infty}^{-\frac{1}{2} c^{-1} \sigma} e^{s} Z([\sigma-c|s|, c|s|] \times \mathrm{d} s), \\
& X_{0}^{\rightarrow}(\sigma)=\int_{-\infty}^{0} e^{s} Z([c|s|, \sigma+c|s|] \times \mathrm{d} s), \\
& X_{0}^{\downarrow}(\sigma)=\int_{-\frac{1}{2} c^{-1} \sigma}^{0} e^{s} Z([c|s|, \sigma-c|s|] \times \mathrm{d} s) .
\end{aligned}
$$

Кроме того, $\left\{X_{0}^{\searrow}(\sigma)\right\}_{\sigma \geqslant 0}$ не зависит от $\left\{X_{0} \rightarrow(\sigma), X_{0}^{\downarrow}(\sigma)\right\}_{\sigma \geqslant 0}$.

Заметим, что динамика относительно $\sigma$ довольно сильно отличается от динамики относительно $t$ (можно сравнить (18) или (19) при $C_{s}(0)=[-c|s|, c|s|]$ с (27)). $\mathrm{B}$ частности, в отличие от $\left\{X_{t}(\sigma)\right\}_{t \in \mathbb{R}}$, процесс $\left\{X_{t}(\sigma)\right\}_{\sigma \in \mathbb{R}}$ не марковский. Однако это различие в сушественной степени зависит от предположения (13). Как мы покажем в разделе 4.3 , на самом деле есть по крайней мере один выбор множеств размаха $A_{t}(\sigma)$, при котором ключевые аспекты динамики оказьваются одними и теми же для обоих процессов, и это связано с тейлоровской гипотезой замороженного потока для турбулентности.

\section{§4. Пространственно-временные процессы СП-типа}

В этом параграфе мы используем результаты $\S \S 2$ и 3 для построения процессов СП-типа, определяемых как экспоненты от процессов $X_{t}(\sigma)$. В контексте процессов СП-типа мы интересуемся главным образом моделированием $n$-точечных корреляций и $n$-точечных корреляторов, которые определены ниже. Благодаря мультипликативной постановке все эти величины можно выразить в терминах k-кумулянтной функции процесса $X_{t}(\sigma)$, что позволяет проводить явные вычисления с помошью функционального уравнения (8). 
Что касается моделирования, в контексте настоящей статьи, то мы главным образом имеем в виду описание стохастического поведения турбулентных полей диссипации энергии $\left\{\varepsilon_{t}(\sigma): \sigma \in \mathscr{S}\right\}_{t \in \mathbb{R}}$. В качестве приложения мы показываем, каким образом СП-поля можно понимать как непрерывные аналоги мультипликативных каскадных процессов при описании турбулентных полей диссипации энергии.

Для простоты на протяжении всего параграфа мы ограничиваемся обсуждением множеств размаха вида

$$
A_{t}(\sigma)=\left\{(\rho, s):-\infty<s<t, \rho \in C_{s-t}(\sigma)\right\}
$$

где

$$
C_{s}(\sigma)=[\sigma-g(s), \sigma+g(s)]
$$

и функция $g(s)$, определенная на $(-\infty, 0]$, неотрицательна и убьвает на $[-T, 0]$, причем $C_{s}(\sigma)=0$ для $s \in(-\infty,-T)$. Как и в $\S 3$, мы определяем $g(s)$ при $s>0$ по правилу $g(s)=g(-s)$.

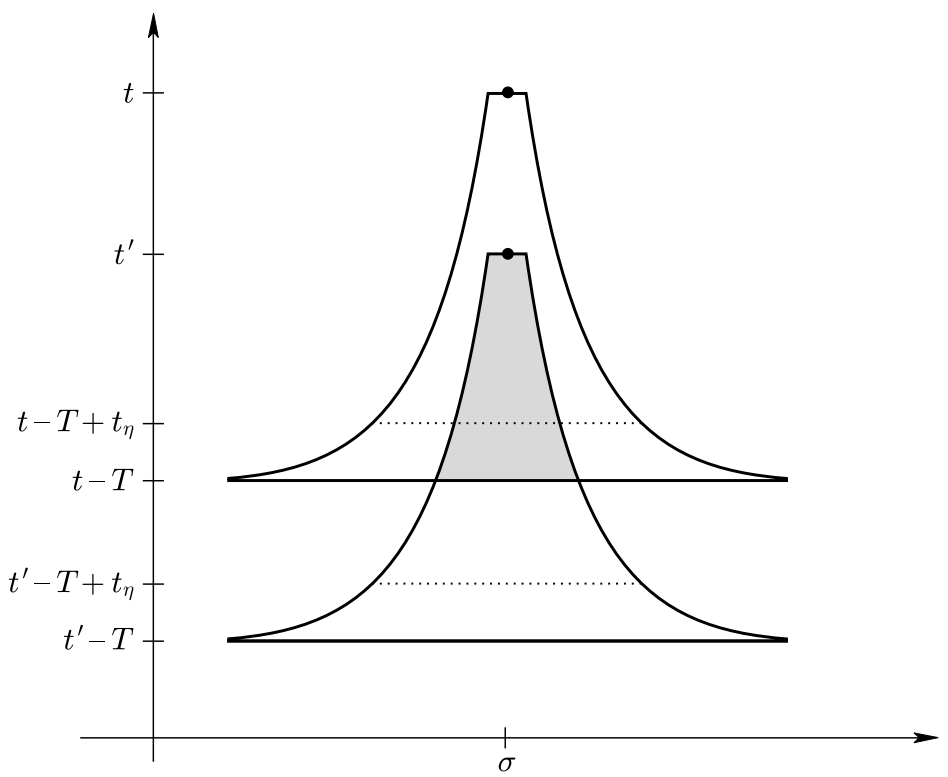

Рис. 5. Иллюстрация перекрытия $A_{t}(\sigma) \cap A_{t^{\prime}}(\sigma)$

Заметим, что в этом случае

$$
\left|A_{t}(\sigma) \cap A_{t^{\prime}}(\sigma)\right|=G\left(\left(T-\left|t-t^{\prime}\right|\right)_{+}\right)
$$

(см. рис. 5), где

$$
G(u)=2 \int_{0}^{u} g(s) \mathrm{d} s .
$$




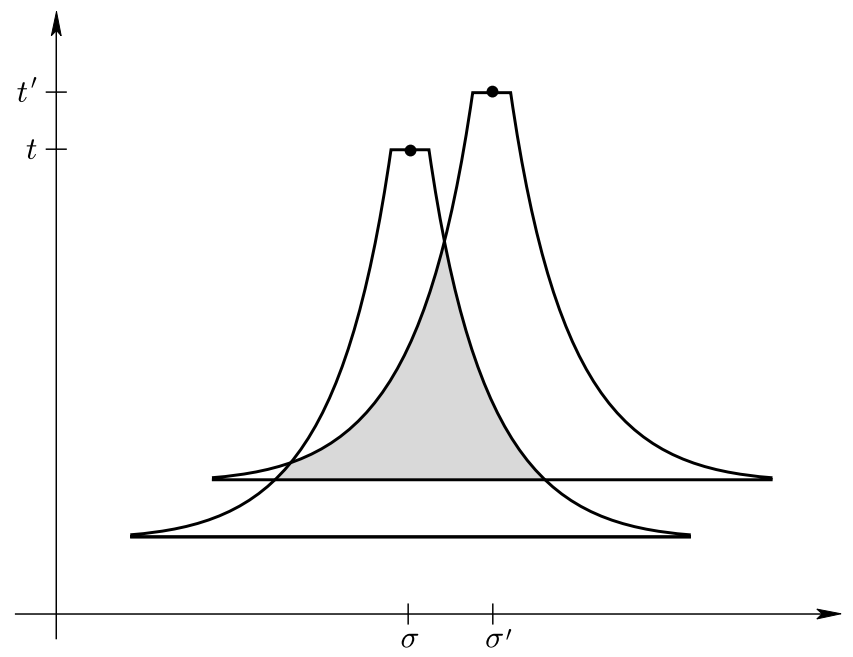

Рис. 6. Иллюстрация перекрытия $A_{t}(\sigma) \cap A_{t^{\prime}}\left(\sigma^{\prime}\right)$

Для сравнения рис. 6 показывает геометрию перекрытия $A_{t}(\sigma) \cap A_{t^{\prime}}\left(\sigma^{\prime}\right)$ при $\sigma \neq \sigma^{\prime}$.

4.1. СП-поля. Процессы, которыми мы сейчас интересуемся, строятся с помошю аддитивных процессов $X_{t}(\sigma)$, определенных, как и вьше, формулой

$$
X_{t}(\sigma)=\int_{-\infty}^{t} \int_{\mathscr{S}} f_{t}(\rho, s ; \sigma) Z(\mathrm{~d} \rho \times \mathrm{d} s),
$$

где $\sigma \in \mathscr{S}, Z$ обозначает базис Леви на $\mathscr{R}=\mathscr{S} \times \mathbb{R}$ и $\mathscr{S}=\mathbb{R}^{d}$. Функции $f$ имеют вид

$$
f_{t}(\rho, s ; \sigma)=h_{t}(\rho, s ; \sigma) \mathbf{1}_{A_{t}(\sigma)}(\rho, \sigma),
$$

где $h$ - специального вида и каждой точке $a=(\sigma, t)$ в $\mathscr{R}$ отвечает некоторое множество размаха $A_{t}(\sigma)$, заданное формулой (31).

В таком случае СП-поле $Y_{t}(\sigma)$ определяется по правилу

$$
Y_{t}(\sigma)=\exp \left\{X_{t}(\sigma)\right\} .
$$

Этот способ задания определяет мультипликативный процесс с независимыми мультипликативньми весами, поскольку базис Леви $Z$ есть независимо рассеянная случайная мера. Поля такого рода называются здесь полями стохастической прерывистости (СП), поскольку мультипликативность позволяет адекватно моделировать прерьвистое поведение. В этом отношении СП-поля могут рассматриваться как динамическое, непрерьвное и однородное обобщение мультипликативных каскадных процессов. Эвристически, это можно видеть из следуюших соображений. В простейшей формулировке, мультипликативньй каскад для величины $\epsilon(\sigma)$ может быть описан формулой

$$
\epsilon(\sigma)=\prod_{j} q_{j}(\sigma)
$$


где мультипликативные веса $q_{j}$ - независимые случайные величины. Они действуют на иерархии шкал $l_{j}$, характеризуемой индексом шкалы $j$, т.е. для любого $j$ сушествует семейство независимых мультипликативных весов $\left\{q_{j}^{i}: i \in \mathbb{N}\right\}$ и связанное с ним семейство множеств $\left\{A_{j}^{i} \in \mathscr{B}(\mathscr{S}): i \in \mathbb{N}\right\}$, образующих разбиение $\mathscr{S}$, причем их евклидовы объемы удовлетворяют условию $l_{j}<l_{j^{\prime}}$ при $j^{\prime}<j$, а $q_{j}(\sigma)=q_{j}^{i}$ для $\sigma \in A_{j}^{i}$. Если мы теперь предположим, что $q_{j}$ положительна для любого $j$, то каскадньй процесс можно переписать в виде

$$
\epsilon(\sigma)=\exp \left\{\sum_{j} \ln q_{j}(\sigma)\right\}
$$

Теперь естественное и динамическое обобщение состоит в том, чтобы уплотнить шкалы, т.е. индекс шкалы $j$ становится непрерывным и отождествляется с величиной $t$, что приводит к структурам, находящимся в рамках постановки (34). Обычное отождествление $t$ с временем устанавливает теперь динамический аспект этого обобщения.

4.2. Корреляторы. Чтобы описать коррелящионную структуру СП-поля, мы используем две родственные величины, полезные для мультипликативных процессов. А именно, определим $n$-точечные корреляиии

$$
m\left(a_{1}, m_{1} ; \ldots ; a_{n}, m_{n}\right)=\mathrm{E}\left\{Y_{t_{1}}\left(\sigma_{1}\right)^{m_{1}} \cdots Y_{t_{n}}\left(\sigma_{n}\right)^{m_{n}}\right\}
$$

и $n$-точечные корреляторьи

$$
c\left(a_{1}, m_{1} ; \ldots ; a_{n}, m_{n}\right)=\frac{m\left(a_{1}, m_{1} ; \ldots ; a_{n}, m_{n}\right)}{m\left(a_{1}, m_{1}\right) \cdots m\left(a_{n}, m_{n}\right)},
$$

предполагая, что соответствуюшие моменты сушествуют и конечны. Здесь $a_{i}=\left(\sigma_{i}, t_{i}\right)$ и $m_{i} \in \mathbb{R}$ при $i=1, \ldots, n$. Это определение корреляторов естественно приспособлено к мультипликативности СП-поля, поскольку оно допускает сокрашение независимых множителей в числителе и знаменателе. В дальнейшем мы всегда предполагаем, что приведенные вьше коррелящи и корреляторы сушествуют. В этом случае можно применить (8) и получить важный результат

$$
m\left(a_{1}, m_{1} ; \ldots ; a_{n}, m_{n}\right)=\exp \left\{\int_{\mathscr{R}} \mathrm{K}\left(\sum_{j=1}^{n} m_{j} f_{t_{j}}\left(a ; \sigma_{j}\right) ; a\right) \mu(\mathrm{d} a)\right\}
$$

где $\mathrm{K}(\theta ; \xi)=\kappa(-i \theta ; \xi)$ - k-кумулянтная функция (мы предполагаем, что она сушествует) случайной величины $Z^{\prime}(\xi)$, определенной в разделе 2.1. Если $Z$ однородна, то мы пишем $\mathrm{K}(\theta)$ вместо $\mathrm{K}(\theta ; \xi)$.

4.3. Масштабирование корреляторов. Здесь масштабирование (скейлинг) некоторой функции $\Psi(x)$ означает, что $\Psi(x)$ демонстрирует степенное поведение $c x^{-\tau}$, где $c$-постоянная и так назьваемьй масштабньй показатель $\tau$ не зависит от $x$. Термин "скейлинг" или "масштабирование" возникает потому, что изменение шкалы $x^{\prime}=$ $k x$ вызывает изменение только величины постоянной $c$. Таким образом, степенное поведение (и $\tau)$ не зависят от масштаба. 
В качестве примера полезности формулы (37) мы построим СП-поле, выражающее масштабные соотношения для $n$-точечных корреляторов. Этот тип корреляционной структуры наблюдается при диссипации энергии в турбулентном потоке и тесно связан с мультифрактальной и прерьвистой природой поля крупнозернистой диссипации энергии (см. [46], [49], [50]). Для краткости мы ограничимся случаем $\mathscr{S}=\mathbb{R}$ и предположим, что базис Леви $Z$ однороден и имеет конечную k-кумулянтную функцию $\mathrm{K}(\theta ; \xi)=\mathrm{K}(\theta)$ для всех $\xi \in \mathscr{S}$ и $\theta \in D(\mathrm{~K})$, где $D(\mathrm{~K})$ - множество значений $\theta$, на которых функция $\mathrm{K}$ определена. Функция $h$ в (33) предполагается постоянной, $h=1$.

В качестве функции $g(s)$ (определяющей множества размаха по формуле $(32)$ ) мы возьмем

$$
g(s)=\frac{1}{2(T+s)} \text { для } s \in\left[t_{\eta}-T, 0\right],
$$

причем на $\left[-T, t_{\eta}-T\right]$ функция остается произвольной, но подчиненной двум условиям: она убьвает на $[-T, 0]$ и число $\left|A_{t}(\sigma)\right|$ конечно, ср. рис. 7.

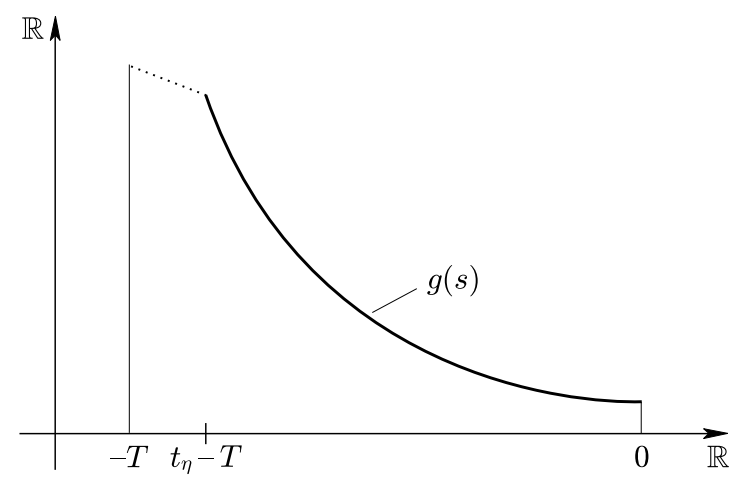

Рис. 7. Иллюстрация: функция $g(t)$

При такой постановке нетрудно видеть (с помощью (36), (37) и рис. 7), что если $m_{1}, m_{2}, m_{1}+m_{2} \in D(\mathrm{~K})$, то

$$
c\left((\sigma, t), m_{1} ;\left(\sigma, t^{\prime}\right), m_{2}\right)=\left(\frac{T}{\left|t-t^{\prime}\right|}\right)^{\mathrm{K}\left(m_{1}+m_{2}\right)-\mathrm{K}\left(m_{1}\right)-\mathrm{K}\left(m_{2}\right)}, \quad\left|t-t^{\prime}\right| \in\left[t_{\eta}, T\right]
$$

а это - масштабирование по $\left|t-t^{\prime}\right|$, и

$c\left((\sigma, t), m_{1} ;\left(\sigma^{\prime}, t\right), m_{2}\right) \propto\left|\sigma-\sigma^{\prime}\right|^{-\left(\mathrm{K}\left(m_{1}+m_{2}\right)-\mathrm{K}\left(m_{1}\right)-\mathrm{K}\left(m_{2}\right)\right)}, \quad\left|\sigma-\sigma^{\prime}\right| \in\left[T^{-1}, t_{\eta}^{-1}\right]$,

а это - масштабирование по $\left|\sigma-\sigma^{\prime}\right|$. Если $m_{1}$ и $m_{2}$ положительны, то масштабный показатель $\tau=\mathrm{K}\left(m_{1}+m_{2}\right)-\mathrm{K}\left(m_{1}\right)-\mathrm{K}\left(m_{2}\right)$ тоже положителен, как показьвает неравенство Минковского. Константа пропорциональности в последнем выражении зависит от $g$. Аналогичные соотношения вьполняются для корреляторов высших порядков, и все они могут быть выражены как функции перекрытий всех участвующих множеств размаха, что, в свою очередь, приводит к масштабируюшим соотношениям. По поводу подробной характеризации корреляторов высших порядков и обобшения 
на случай $S=\mathbb{R}^{d}$ мы отсылаем к работам [46], [49], [50], [8]. Здесь мы формулируем только основные результаты. Для возрастающей последовательности $\left\{t_{j}, 1 \leqslant j \leqslant n\right\}$ с $a_{i}=\left(\sigma, t_{i}\right)$ получаем

$$
c\left(a_{1}, m_{1} ; \ldots ; a_{n}, m_{n}\right) \propto \prod_{j=1}^{n-1} \prod_{k=j+1}^{n}\left(t_{k}-t_{k-j}\right)^{-\tau\left[m_{k-j}, \ldots, m_{k}\right]}
$$

для временных корреляторов, где показатели $\tau\left[m_{k-j}, \ldots, m_{k}\right]$ определяются формулой

$$
\tau\left[m_{1}, \ldots, m_{j}\right]=\mathrm{K}\left(\sum_{i=1}^{j-2} m_{i}\right)+\mathrm{K}\left(\sum_{i=1}^{j} m_{i}\right)-2 \mathrm{~K}\left(\sum_{i=1}^{j-1} m_{i}\right) .
$$

Эти показатели могут быть как положительны, так и отрищательны, в зависимости от $j$ и от порядков $m$. Однако при $j=2$ они строго положительны для всех положительных $m$.

Следующий шаг моделирования состоит в таком выборе $\mathrm{k}$-кумулянтной функции $\mathrm{K}$, чтобы получаемые с ее помошью значения показателей $\tau\left[m_{1}, \ldots, m_{j}\right]$, даваемые формулой (40), соответствовали эмпирически определяемым значениям этих показателей с наибольшей возможной точностью. Мы обсуждаем этот вопрос в [8] (см. также [50]).

Для пространственных корреляторов мы получаем похожее соотношение

$$
c\left(a_{1}, m_{1} ; \ldots ; a_{n}, m_{n}\right) \propto \prod_{j=1}^{n-1} \prod_{k=j+1}^{n}\left(\sigma_{k}-\sigma_{k-j}\right)^{-\tau\left[m_{k-j}, \ldots, m_{k}\right]}
$$

для возрастающей последовательности $\left\{\sigma_{j}, 1 \leqslant j \leqslant n\right\}$ с $a_{i}=\left(\sigma_{i}, t\right)$. Заметим, что масштабируюшие показатели $\tau$ - одни и те же в обоих случаях. Однако в [46] показано, как построить СП-поле, для которого эти показатели различны.

Для дальнейшей иллюстрации этих масштабируюших соотношений мы приведем следующее выражение для временно́го коррелятора с $n=3$ :

$c\left(a_{1}, m_{1} ; a_{2}, m_{2} ; a_{3}, m_{3}\right) \propto\left(t_{2}-t_{1}\right)^{-\tau\left[m_{1}, m_{2}\right]}\left(t_{3}-t_{2}\right)^{-\tau\left[m_{2}, m_{3}\right]}\left(t_{3}-t_{1}\right)^{-\tau\left[m_{1}, m_{2}, m_{3}\right]}$.

Аргументы масштабируюших показателей $\tau$ отражают гнездовую структуру различных перекрытий. Здесь точки $a_{1}, a_{2}$ и $a_{2}, a_{3}$ непосредственно соседствуют, а точки $a_{1}, a_{3}$ одновременно соседствуют с некоторой точкой.

Можно также мотивировать вид (38) функции $g(t)$ с помошью тейлоровской гипотезы замороженного потока (по поводу обсуждения этой гипотезы см. [25]). Она утверждает, что пространственная вариация диссипации энергии может быть выражена в терминах временно́й вариации с помошью средней скорости потока $v_{0}$, если относительные флуктуации около $v_{0}$ малы. Можно вывести (38) с помошью этого предположения и определения множества размаха $A_{t}(\sigma)$ как множества, ограниченного с помошью функции $g$, как в (32). Более точно, формула (38) следует из предположения, что процессы $\left\{Y_{t}(\sigma)\right\}_{t \in \mathbb{R}}$ и $\left\{Y_{t}(\sigma)\right\}_{\sigma \in \mathbb{R}}$ имеют одинаковые дву хточечные корреляторы. (Подробности можно найти в статьях [47] и [8].) 
4.4. Дальнейшее моделирование двухточечных корреляторов. В предыдушем разделе мы использовали СП-поле для моделирования масштабирования двухточечных корреляторов с помошью специального выбора функции $g(s)$ в $(38)$, и поэтому выбора соответствуюших множеств размаха $A_{t}(\sigma)$. В дальнейшем мы приведем некоторые примеры того, что можно моделировать, используя больше степеней свободы, входящих в определение СП-поля $Y_{t}(\sigma)$.

Чтобы выразить идеи ясно, мы примем постановку задачи из раздела 4.3 и выберем функцию $g(s)$ так, чтобы моделировать формы, отличные от масштабирования двухточечных корреляторов. Приведенные здесь идеи могут также служить отправной точкой моделирования пространственно-временных корреляторов высших порядков с помощью всех степеней свободы, входящих в общее определение СП-поля. Но сейчас мы рассматриваем только двухточечные корреляторы и сосредоточиваемся на степени свободы в выборе множеств размаха $A_{t}(\sigma)$ в случае $S=\mathbb{R}$ и для однородного базиса Леви $Z$. Мы также предполагаем, что рассматриваемое СП-поле однородно, что достигается инвариантностью множеств размаха относительно формы, т.е. условием $A_{t}(\sigma)=(\sigma, t)+A$. Исходной точкой является уравнение $(37)$, которое упрошается в рассматриваемом случае следуюшим образом:

$$
c\left((\sigma, t), 1 ;\left(\sigma, t^{\prime}\right), 1\right)=c\left(\left|t-t^{\prime}\right|\right)=e^{\left|A_{t}(\sigma) \cap A_{t^{\prime}}(\sigma)\right|(\mathrm{K}(2)-2 \mathrm{~K}(1))},
$$

где разность $\mathrm{K}(2)-2 \mathrm{~K}(1)$ положительна и $c\left(\left|t-t^{\prime}\right|\right)$ - стенографическое выражение. Иначе говоря,

$$
\ln c(u)=(\mathrm{K}(2)-2 \mathrm{~K}(1)) G(T-u)
$$

при $u \in(0, T]$. Итак, если $g$ непрерьвна на $[-T, 0]$, то

$$
g(u)=-\frac{1}{\mathrm{~K}(2)-2 \mathrm{~K}(1)} \frac{c^{\prime}(T-u)}{c(T-u)}
$$

при $u \in(0, T]$. Это указьвает, как выбрать $g$, чтобы получить желаемое поведение двухточечной корреляторной функции $c$.

ПримеР 4.1 (экспоненциальное убывание). Предположим, что мы хотим моделировать процесс с корреляторной функцией, равной $c(t)=1+e^{-\lambda t}$ при $0<t<T$ и $c(t)=1$ при $t>T$, где $\lambda>0$. Тогда из (44) следует, что

$$
g(u)=-\frac{1}{\mathrm{~K}(2)-2 \mathrm{~K}(1)} \frac{\lambda e^{-\lambda(T-u)}}{1+e^{-\lambda(T-u)}},
$$

и эта функция определяет множество размаха $A_{t}(\sigma)$ по формулам (31) и (32). В физике экспоненциальное убывание есть часто применяемая модель близкодействующей коррелящии в равновесных системах.

ПРимеР 4.2 (степенное убьвание). Предположим, что мы хотим моделировать процесс с корреляторной функцией, равной $c(t)=1+\left(t+t_{0}\right)^{-\alpha}$, где $\alpha>0$ и $t_{0}>0$. Тогда

$$
g(u)=-\frac{1}{\mathrm{~K}(2)-2 \mathrm{~K}(1)} \frac{\alpha\left(T-u+t_{0}\right)^{-\alpha-1}}{\left(1+\left(T-u+t_{0}\right)^{-\alpha}\right)} .
$$

Этот пример может рассматриваться, как приближение к масштабированию двухточечных корреляторов для $t \gg t_{0}$. 
4.5. Динамическое среднедисперсное моделирование.

Общие замечания. В этом разделе мы рассматриваем главным образом нестационарные СП-поля. В частности, мы заинтересованы в построении СП-полей с данной временной эволюцией процесса среднего и процесса дисперсии, оставляя в стороне обсуждение возможных корреляций. Таким образом, цель этого раздела - моделирование временной эволюции первого и второго моментов

$$
\begin{aligned}
\mathrm{E}\left\{Y_{t}(\sigma)\right\} & =\exp \left\{\int_{\mathscr{R}} \mathrm{K}\left(h_{t}(a ; \sigma) 1_{A_{t}(\sigma)} ; a\right) \mu(\mathrm{d} a)\right\} \equiv \mu_{1}(t), \\
\mathrm{E}\left\{\left(Y_{t}(\sigma)\right)^{2}\right\} & =\exp \left\{\int_{\mathscr{R}} \mathrm{K}\left(2 h_{t}(a ; \sigma) 1_{A_{t}(\sigma)} ; a\right) \mu(\mathrm{d} a)\right\} \equiv \mu_{2}(t),
\end{aligned}
$$

которые предполагаются независимыми от $\sigma$. Основное предположение снова состоит в наличии факторизуемого базиса Леви $Z$, откуда следует, что величина $\mathrm{K}(\zeta ; a)$ не зависит от $a \in \mathscr{R}$. Однако допускается явная зависимость величины $A_{t}(\sigma)$ от $t$ и непостоянство функций $h_{t}(a ; \sigma)$. Это дает достаточную свободу для независимого моделирования моментов $\mu_{1}(t)$ и $\mu_{2}(t)$.

Процесс дисперсии с постоянным средним. Простейшая ситуация связана с постоянным средним $\mu_{1}(t)=1$ и переменным вторым моментом $\mu_{2}(t)$. Первое условие достигается, если просто положить $h_{t}(a, \sigma)=c_{h}=$ const таким образом, чтобы $\mathrm{K}\left(c_{h}\right)=0$ и $\mathrm{K}\left(2 c_{h}\right) \neq 0$, если такая постоянная существует. Это требует, чтобы k-кумулянтная функция K существовала в окрестности точки 0. Тогда средние становятся независимы от $A_{t}(\sigma)$, в то время как

$$
\ln \mu_{2}(t)=\mathrm{K}\left(2 c_{h}\right) \mu\left(A_{t}(\sigma)\right),
$$

и мы, вообще говоря, можем выбрать множества $A_{t}(\sigma)$ и меру $\mu$ так, чтобы моделировать $\mu_{2}(t)$.

ПримеР 4.3 (возрастаюший второй момент (I)). Для простоты положим $\mathscr{R}=\mathbb{R}^{4}$ и в качестве $\mu$ возьмем лебегову меру. Предположим, что мы хотим найти дифференцируемую функцию $\mu_{2}(t)$ с $\mu_{2}^{\prime}(t)>0$ такую, что $\mu_{2}(t) \rightarrow 1$ при $t \rightarrow-\infty$. Пусть $B(r)$ обозначает шар радиуса $r$. Предположим, что $A_{t}(\sigma)$ имеет вид

$$
A_{t}(\sigma)=\{(\sigma, s)+B(r(s)): s \leqslant t\}
$$

для некоторой функщии $r(s) \geqslant 0$.

Тогда

$$
\mu\left(A_{t}(\sigma)\right)=\int_{-\infty}^{t} \mathrm{~d} s \int_{(\sigma, s)+B(r(s))} \mathrm{d} \rho
$$

и, следовательно, полагая

$$
r(t)=\left(\frac{3 \mu_{2}^{\prime}(t)}{4 \pi \mathrm{K}\left(2 c_{h}\right) \mu_{2}(t)}\right)^{1 / 3}
$$

мы видим, что (46) выполнено. 
Например, мы можем взять $\mu_{2}(t)=1+e^{t}$ и

$$
Y_{t}(\sigma)=\exp \left\{\int_{-\infty}^{t} \int_{\left(\sigma, t^{\prime}\right)+B\left(r\left(t^{\prime}\right)\right)} Z\left(\mathrm{~d} a^{\prime}\right)\right\},
$$

где базис Леви $Z$ определен однородным, причем $Z^{\prime}$ распределен в соответствии с устойчивым законом $S_{\alpha}(\sigma, \beta, \mu)^{4}$ с $\alpha \neq 1, \beta=-1$ и

$$
\mu=\frac{\sigma^{\alpha}}{\cos \left(\frac{\pi \alpha}{2}\right)} .
$$

В этом случае k-кумулянтная функция $Z^{\prime}$ равна функции

$$
\mathrm{K}(\theta)=\mu\left(\theta-\theta^{\alpha}\right)
$$

с двумя нулями $\theta=0$ и $\theta=1$. Таким образом, мы полагаем $c_{h}=1$. В соответствии с (49) мы выбираем

$$
r(t)=\frac{3 e^{t}}{4 \pi \mathrm{K}(2)\left(1+e^{t}\right)}
$$

и получаем $\mu_{1}(t)=1$ и $\mu_{2}(t)=1+e^{t}$.

ПРИмеР 4.4 (возрастающий второй момент (II)). Здесь мы используем однородньй НОГ базис Леви вместо устойчивого базиса Леви, использованного в предыдущем примере. Теперь k-кумулянтная функция равна

$$
\mathrm{K}(\theta)=\delta\left\{\sqrt{\alpha^{2}-\beta^{2}}-\sqrt{\alpha^{2}-(\beta+\theta)^{2}}\right\}+\mu \theta
$$

где $\mu \in \mathbb{R}, \delta \in \mathbb{R}_{+}$и $0 \leqslant|\beta|<\alpha$. Если мы определим постоянную $c_{h}$ формулой $\left(\beta+c_{h}\right)^{2}=\alpha^{2}$ и положим

$$
\delta=\frac{-\mu c_{h}}{\sqrt{\alpha^{2}-\beta^{2}}}
$$

то мы получим $\mathrm{K}\left(c_{h}\right)=0$, и если $Y_{t}(\sigma)$ определен формулой $(50)$ при

$$
r(t)=\frac{3 e^{t}}{4 \pi \mathrm{K}\left(2 c_{h}\right)\left(1+e^{t}\right)},
$$

то $\mu_{1}(t)=1$ и $\mu_{2}(t)=1+e^{t}$.

\footnotetext{
${ }^{4}$ Здесь мы используем обозначения, введенные в [43], в соответствии с которыми $S_{\alpha}(\sigma, \beta, \mu)-$ безгранично делимьй закон с с-кумулянтной функцией

$$
\mathrm{C}\{\theta \ddagger X\}=\left\{\begin{array}{lc}
\exp \left\{-\sigma^{\alpha}|\theta|^{\alpha}\left(1-i \beta\left(\operatorname{sign}(\theta) \operatorname{tg}\left(\frac{\pi \alpha}{2}\right)\right)\right)+i \mu \theta\right\} & (\alpha \neq 1), \\
\exp \left\{-\sigma|\theta|\left(1+i \beta \frac{2}{\pi}(\operatorname{sign}(\theta) \ln (|\theta|))\right)+i \mu \theta\right\} & (\alpha=1) .
\end{array}\right.
$$
}


ПримеР 4.5 (произвольно меняющийся второй момент). Положим

$$
Y_{t}(\sigma)=\exp \left\{\int_{(\sigma, t)+B(r(t))} Z\left(\mathrm{~d} a^{\prime}\right)\right\},
$$

где $Z$ снова однороден. Если взять

$$
r(t)=\left(\frac{3 \ln \mu_{2}(t)}{4 \pi \mathrm{K}(2)}\right)^{1 / 3},
$$

то $\mathrm{E}\left\{\left(Y_{t}(\sigma)\right)^{2}\right\}=\mu_{2}(t)$ и $\mathrm{E}\left\{Y_{t}(\sigma)\right\}=1$, где $\mu_{2}(t) \geqslant 1$ может быть выбран произвольно.

Процессы среднего и дисперсии. Мы теперь сосредоточимся на СП-полях, для которых оба момента $\mu_{1}(t)$ и $\mu_{2}(t)$ меняются вместе $\mathrm{c} t$. В отличие от предыдущего, мы теперь рассматриваем случай, когда функция $h_{t}(a ; \sigma)$ меняется с изменением $t$, но не меняется, когда меняются $a$ и $\sigma$. Соответственно мы записываем эту функцию как $h_{t}$. Тогда (45) и (46) принимают вид

$$
\begin{aligned}
\mathrm{E}\left\{Y_{t}(\sigma)\right\} & =\exp \left\{\mu\left(A_{t}(\sigma)\right) \mathrm{K}\left(h_{t} 1_{A_{t}(\sigma)}\right)\right\} \equiv \mu_{1}(t), \\
\mathrm{E}\left\{\left(Y_{t}(\sigma)\right)^{2}\right\} & =\exp \left\{\mu\left(A_{t}(\sigma)\right) \mathrm{K}\left(2 h_{t} 1_{A_{t}(\sigma)}\right)\right\} \equiv \mu_{2}(t) .
\end{aligned}
$$

Чтобы решить эти два уравнения, мы имеем в своем распоряжении три величины, а именно, $\mathrm{K}, h$ и $\mu\left(A_{t}(\sigma)\right)$. Поэтому мы можем ограничиться выбором k-кумулянтной функции $\mathrm{K}$ заранее некоторым подходяшим образом и затем решать (51) и (52) относительно $h_{t}$ и $\mu\left(A_{t}(\sigma)\right)$. Заметим здесь, что $A_{t}(\sigma)$ все еше произвольны и только мера $\mu\left(A_{t}(\sigma)\right)$ определена. Таким образом, еше остается некоторая свобода в моделировании коррелящионной структуры СП-поля $Y_{t}(\sigma)$.

ПримеР 4.6. Особенно простой пример дается однородным устойчивым базисом Леви на $\mathscr{R}=\mathbb{R}^{4}$, определенным в примере 4.3. Из уравнений (51) и (52) следует, что

$$
q_{t} \equiv \frac{\ln \mu_{2}(t)}{\ln \mu_{1}(t)}=\frac{\mathrm{K}\left(2 h_{t}\right)}{\mathrm{K}\left(h_{t}\right)}=\frac{2 h_{t}-\left(2 h_{t}\right)^{\alpha}}{h_{t}-h_{t}^{\alpha}} .
$$

Предполагая теперь, что $q_{t}>2^{\alpha}$ для всех $t$ (что тривиально вьполняется при $\alpha<1$ ), мы получаем

$$
h_{t}=\left(\frac{q_{t}-2}{q_{t}-2^{\alpha}}\right)^{1 /(\alpha-1)} .
$$

Наконец, положим

$$
r(t)=\left(\frac{3 \ln \mu_{1}(t)}{4 \pi \mathrm{K}\left(h_{t}\right)}\right)^{1 / 3}
$$

и определим множества размаха $A_{t}(\sigma)=(\sigma, t)+B_{r(t)}$ и СП-поле $Y_{t}(\sigma)$ - как в примеpe 4.3. В этой постановке уравнения (51) и (52) вьполняются для произвольных функший $\mu_{1}(t)$ и $\mu_{2}(t)$, если $q_{t}>2^{\alpha}$. Частньй пример дается формулой $\mu_{2}(t)=\mu_{1}(t)^{\tau}+$ $\mu_{1}(t)^{2}$ с произвольньм $\tau$. Этот тип соотношения между первым и вторым моментами является определяющей особенностью экспоненциального семейства моделей Твиди (ср., например, [28]). 


\section{$\S 5$. Выводы}

Мы обсудили некоторые пространственно-временные модели $X_{t}(\sigma)$ и $Y_{t}(\sigma)=$ $\exp \left\{X_{t}(\sigma)\right\}$, где модели $X_{t}(\sigma)$ определяются в терминах интегралов по базисам Леви. Нашей основной целью было построение реалистических динамических моделей, непрерывных по пространству и времени, для полей диссипации энергии $\epsilon_{t}(\sigma)$ турбулентности при большом числе Рейнольдса, в соответствии с недавними успехами (в частности, формулировками в терминах случайных каскадов) исходной феноменологической теории однородной и изотропной турбулентности, принадлежащей Колмогорову. (Мы также кратко обсуждаем применимость результатов, полученных при пространственно-временном моделировании, в частности, с помошью анализа изображений.) В отличие от моделей дискретных случайных каскадов, рассматривавшихся в [27], [22], [24] и [29], предлагаемые здесь модели непрерывны и однородны; в частности, мы уточняем, как одна из рассматриваемых моделей оказывается в полном согласии с тейлоровской гипотезой замороженного потока.

Мы надеемся в дальнейшей работе извлечь гораздо больше сведений из возможностей рамок настоящей работы для более подробного, ясного и цельного моделирования турбулентных полей, с пристальным вниманием к недавним экспериментальньм свидетельствам.

\section{СПИСОК ЛИТЕРАТУРЫ}

[1] O. E. Barndorff-Nielsen. Probability and statistics; selfdecomposability, finance and turbulence // Proceedings of the Conference "Probability towards 2000" (New York, 1995) / ed. L. Accardi, C. C. Heyde. New York: Springer-Verlag, 1998. P. 47-57. (Lecture Notes in Statist. V. 128.)

[2] O. E. Barndorff-Nielsen. Processes of normal inverse Gaussian type // Finance Stoch. 1998. V. 2. № 1. P. 41-68.

[3] O. E. Barndorff-Nielsen. Superposition of Ornstein-Uhlenbeck type processes // Teoрия вероятн. и ее примен. 2001. Т. 45. № 2. С. 289-311.

[4] O. E. Barndorff-Nielsen. Modelling by Lévy processes // Selected Proceedings of the Symposium on Inference for Stochastic Processes (Athens, 2000) / ed. I. V. Basawa, C.C. Heyde, R. L. Taylor. Beachwood: Inst. Math. Statist., 2001. P. 25-31. (IMS Lecture Notes Monogr. Ser. V. 37.)

[5] O. E. Barndorff-Nielsen, J. L. Jensen, M. Sørensen. Parametric modelling of turbulence // Philos. Trans. Roy. Soc. London A. 1990. V. 332. P. 439-455.

[6] O. E. Barndorff-Nielsen, J.L. Jensen, M. Sørensen. A statistical model for the streamwise component of a turbulent velocity field // Ann. Geophys. 1993. V. 11. P. 99-103.

[7] O. E. Barndorff-Nielsen, J. Pedersen, K. Sato. Multivariate subordination, self-decomposability and stability // Adv. in Appl. Probab. 2001. V. 33. № 1. P. 160-187.

[8] O. E. Barndorff-Nielsen, J. Schmiegel. Lévy-based spatial-temporal modelling // In preparation, 2003.

[9] O. E. Barndorff-Nielsen, N. Shephard. Modelling by Lévy processes for financial econometrics // Lévy Processes - Theory and Applications / ed. O.E. Barndorff-Nielsen, T. Mikosch, S. Resnick. Boston: Birkhäuser, 2001. P. 283-318.

[10] O. E. Barndorff-Nielsen, N. Shephard. Non-Gaussian Ornstein-Uhlenbeck-based models and some of their uses in financial economics // J. R. Stat. Soc. Ser. B Stat. Methodol. 2001. V. 63. № 2. P. 167-241.

[11] O. E. Barndorff-Nielsen, N. Shephard. Normal modified stable processes // Theory Probab. Math. Statist. 2001. №65. P. 1-20. 
[12] O. E. Barndorff-Nielsen, N. Shephard. Integrated OU processes and non-Gaussian OU-based stochastic volatility models // Scand. J. Statist. 2003. V. 30. № 2. P. 277-295.

[13] O. E. Barndorff-Nielsen, N. Shephard. Financial Volatility: Stochastic Volatility and Lévy Based Models. Cambridge: Cambridge Univ. Press, 2004 (to appear).

[14] O. E. Barndorff-Nielsen, S. Thorbjørnsen. A connection between classical and free infinite divisibility // Research Report 2003-7. Aarhus: Univ. of Aarhus, MaPhySto, 2003.

[15] T. Bohr, M. H. Jensen, G. Paladin, A. Vulpiani. Dynamical Systems Approach to Turbulence. Cambridge: Cambridge Univ. Press, 1998. (Cambridge Nonlinear Science Series. V. 8.)

[16] A. Brix. Spatial and Spatio-Temporal Models for Weed Abundance // Ph.D. Thesis. Copenhagen: Royal Veterinary and Agricultural University, 1998.

[17] A. Brix. Generalized gamma measures and shot-noise Cox processes // Adv. in Appl. Probab. 1999. V. 31. № 4. P. 929-953.

[18] A. Brix, J. Chad ouf. Spatio-temporal modelling of weeds by shot-noise $G$ Cox processes // Biom. J. 2002. V. 44. № 1. P. 83-99.

[19] A. Brix, P. J. Diggle. Spatiotemporal prediction for log-Gaussian Cox processes // J. R. Stat. Soc. Ser. B Stat. Methodol. 2001. V. 63. № 4. P. 823-841.

[20] M. Çag̃lar, E. Çinlar. Lyapunov exponents of Poisson shot-noise velocity fields // Stochastic Process. Appl. 2001. V. 94. № 1. P. 29-49.

[21] P. Carmona, F. Petit, M. Yor. Exponential functionals of Lévy processes // Lévy Processes - Theory and Applications / ed. O.E. Barndorff-Nielsen, T. Mikosch, S. Resnick. Boston: Birkhäuser, 2001. P. 41-55.

[22] J. Cleve, M. Greiner. The Markovian metamorphosis of a simple turbulent cascade model // Phys. Lett. A. 2000. V. 273. № 1-2. P. 104-108.

[23] D. Duffie, D. Filipović, W. Schachermayer. Affine processes and applications in finance // Ann. Appl. Probab. 2003. V. 13. № 3. P. 984-1053.

[24] H. C. Eggers, T. Dziekan, M. Greiner. Translationally invariant cumulants in energy cascade models of turbulence // Phys. Lett. A. 2001. V. 281. № 4. P. 249-255.

[25] U. Frisch. Turbulence. The Legacy of A. N. Kolmogorov. Cambridge: Cambridge Univ. Press, 1995.

[26] S. Ghashgaie, W. Breymann, J. Peinke, P. Talkner, Y. Dodge. Turbulent cascades in foreign exchange markets // Nature. 1996. V. 381. P. 767-770.

[27] M. Greiner. Turbulent random multiplicative branching processes // Lectures on Multiscale and Multiplicative Processes in Fluid Flows / ed. E. Waymire et al. Aarhus: MaPhySto, Univ. of Aarhus, 2002. P. 81-88. (MaPhySto Lecture Notes № 11.)

[28] B. Jørgensen. The Theory of Dispersion Models. London: Chapman \& Hall, 1997.

[29] B. Jouault, M. Greiner, P. Lipa. Fix-point multiplier distributions in discrete turbulent cascade models // Phys. D. 2000. V. 136. P. 125-144.

[30] O. Kallenberg. Random Measures. Berlin: Akademie-Verlag, 1986.

[31] А. Н. Колмогоров. Локалшная структура турбулентности в несжимаемой вязкой жидкости при очень больших числах Рейнольдса // Докл. АН СССР. 1941. Т. 30. № 4 . C. 299-303.

[32] А.Н. Колмогоров. Рассеяние энергии при локально изотропной турбулентности // Докл. АН СССР. 1941. Т. 32. № 1. С. 19-21.

[33] А.Н.Колмогоров. Уточнение представлений о локалњной структуре турбулентности в несжимаемой вязкой жидкости при больших числах Рейнолшдса // Mécanique de la turbulence: Actes du Colloque International du CNRS 1962. P. 447-451; А. Н. Колмогоров. Избранные труды. Математика и механика. М.: Наука, 1985.

[34] S. Kwapień, W. A. Woyczyński. Random Series and Stochastic Integrals: Single and Multiple. Boston: Birkhäuser, 1992.

[35] C. Meneveau, K. R. Sreenivasan. The multifractal nature of turbulent energy dissipation // J. Fluid Mech. 1991. V. 224. P. 429-484.

[36] J. Møller. Shot noise Cox processes // Research Report 2002-18. Aarhus: MaPhySto, Univ. of Aarhus, 2002. 
[37] J. Møller, A. R. Syversveen, R. P. Waagepetersen. Log Gaussian Cox processes // Scand. J. Statist. 1998. V. 25. № 3. P. 451-482.

[38] А. С. Монин, А. М. Яглом. Статистическая гидромеханика. Т. 1, 2. М.: Наука, 1965, 1967.

[39] E. Nicolato, E. Venardos. Option pricing in stochastic volatility models of the Ornstein-Uhlenbeck type // Math. Finance. 2003. V. 13. № 4. P. 445-466.

[40] A. M. Obukhov. Some specific features of atmospheric turbulence // J. Fluid Mech. 1962. V. 13. P. 77-81.

[41] J. Pedersen. The Lévy-Ito decomposition of an independently scattered random measure // Research Report 2003-2. Aarhus: MaPhySto, Univ. of Aarhus, 2003.

[42] B. Rüdiger. Stochastic integration with respect to compensated Poisson random measures on separable Banach spaces // Research Report, SFB 611. Bonn: Universität Bonn, 2003.

[43] G. Samorodnitsky, M. Taqqu. Stable non-Gaussian Random Processes. New York: Chapman \& Hall, 1994.

[44] K. Sato. Lévy Processes and Infinitely Divisible Distributions. Cambridge: Cambridge Univ. Press, 1999.

[45] W. Schoutens. Lévy Processes in Finance. New York: Wiley, 2003.

[46] J. Schmiegel. Ein dynamischer Prozess für die statistische Beschreibung der Energiedissipation in der vollentwickelten Turbulenz // Dissertation TU Dresden, Germany, 2002.

[47] J. Schmiegel. Some remarks on Taylor's Frozen Flow Hypothesis // Research Report. Aarhus: MaPhySto, Univ. of Aarhus, 2003.

[48] J. Schmiegel, T. Dziekan, J. Cleve, B. Jouault, M. Greiner. Scaling functions in a random multiplicative energy-cascade model of turbulence // Phys. Rev. E. 2002 (to appear).

[49] J. Schmiegel, H. C. Eggers, M. Greiner. Dynamical random multiplicative cascade model in 1+1 dimensions // Preprint cond-mat/0106347, 2001.

[50] J. Schmiegel, H. C. Eggers, M. Greiner. A spatio-temporal and causal multifractal stochastic process // Phys. A. 2003 (to appear).

[51] A. N. Shiryaev. Kolmogorov and the Turbulence. Aarhus: MaPhySto, Univ. of Aarhus., 1999. (Miscellanea. № 12.)

[52] K. R. Sreenivasan, R. A. Antonia. The phenomenology of small-scale turbulence // Annu. Rev. Fluid Mech. 1997. V. 29. № 2. P. 435-472.

[53] R. L. Wolpert, K. Ickstadt. Poisson/gamma random field models for spatial statistics // Biometrika. 1998. V. 85. № 2. P. 251-267. 